\title{
On Proper Labellings of Graphs with Minimum Label Sum
}

\author{
Julien Bensmail, Foivos Fioravantes, Nicolas Nisse*
}

January 24, 2020

\begin{abstract}
The 1-2-3 Conjecture, raised by Karoński, Łuczak and Thomason, states that almost every graph $G$ admits a proper 3-labelling, i.e., a labelling of the edges with 1,2,3 such that no two adjacent vertices are incident to the same sum of labels. Another interpretation of this conjecture, that may be attributed to Chartrand et al., is that almost every graph $G$ can be turned into a locally irregular multigraph $M$, i.e., with no two adjacent vertices of the same degree, by replacing each of its edges by at most three parallel edges. In other words, for almost every graph $G$ there should be a locally irregular multigraph $M$ with the same adjacencies and having a relatively small number of edges. The 1-2-3 Conjecture, if true, would indeed imply that there is such an $M$ with $|E(M)| \leq 3|E(G)|$.

In this work, we study proper labellings of graphs with the extra requirement that the sum of assigned labels must be as small as possible. In other words, given a graph $G$, we are looking for a locally irregular multigraph $M^{*}$ with the smallest number of edges possible that can be obtained from $G$ by multiplying edges. This problem is actually quite different from the 1-2-3 Conjecture, as we prove that there is no absolute constant $k$ such that $M^{*}$ can always be obtained from $G$ by replacing each edge with at most $k$ parallel edges.

We investigate several aspects of this problem, covering algorithmic and combinatorial aspects. In particular, we prove that the problem of designing proper labellings with minimum label sum is $\mathcal{N} \mathcal{P}$-hard in general, but solvable in polynomial time for graphs with bounded treewidth. We also conjecture that for all almost every graph $G$ there should be a proper labelling with label sum at most $2|E(G)|$, which we verify for several classes of graphs.
\end{abstract}

\section{Introduction}

In this work we study properties of some distinguishing labellings of graphs. We begin by giving some useful definitions and notations. Let $G=(V, E)$ be a graph. For a $v \in V$, let $d(v)$ denote the degree of $v$, that is the number of edges that are incident to $v$. We say that $G$ is regular if for each $u, v \in V$, we have $d(u)=d(v)$. It is well known that there exists no non-trivial simple graph that is totally irregular, meaning a simple graph for which all vertices have different degrees. This however is not true for multigraphs, where multiple edges between two adjacent vertices are allowed. A graph $G$ is called locally irregular if for each $u v \in E$, we have $d(u) \neq d(v)$. Finally, $G$ is called nice if it does not contain a connected component isomorphic to $K_{2}$, the complete graph with two vertices. Throughout this work, we focus only on nice graphs.

A function $\ell: E \mapsto\{1, \ldots, k\}$, is called a $k$-labelling of $G$. By a distinguishing labelling we mean a labelling $\ell$ that allows us to distinguish the vertices of $G$ accordingly to some vertex parameter computed from $\ell$. Note that this can equivalently be seen through the scope of graph colouring. In this work, we are more particularly interested in proper labellings, which are defined as follows. For any $v \in V$, let us denote by $c_{\ell}(v)$ the colour of $v$ that is induced by $\ell$, being the sum of labels assigned to the edges incident to $v$. That is,

$$
c_{\ell}(v):=\sum_{u \in N(v)} \ell(v u) .
$$

We say that $\ell$ is proper if the resulting $c_{\ell}$ is a proper vertex-colouring of $G$, i.e., for every edge $u v \in E$ we have $c_{\ell}(u) \neq c_{\ell}(v)$. In this work, every labelling is assumed to be proper unless stated

\footnotetext{
*Université Côte d'Azur, CNRS, Inria, I3S, France
} 
otherwise. It is clear that for each not necessarily proper labelling $\ell$ of $G$ and for each $v \in V$, we have $c_{\ell}(v) \geq d(v)$ with $c_{\ell}(v)=d(v)$ if and only if all edges incident to $v$ are assigned label 1 . It follows that $G$ admits a proper 1-labelling if and only if $G$ is locally irregular.

The connection between the labellings of a graph $G$ and its "regularity" was already explored by several authors, in particular through the notion of irregularity strength of graphs, introduced by Chartrand et al. in [9]. The irregularity strength $s(G)$ of $G$ is defined as the smallest $k$ such that $G$ admits a $k$-labelling $\ell$ with the property that for every two $u, v \in V$ (not necessarily adjacent), we have $c_{\ell}(u) \neq c_{\ell}(v)$. The main point for studying such labellings $\ell$, is that if, from $G$, we replace each edge $e$ by $\ell(e)$ parallel edges, then we get a multigraph $M$ that is totally irregular. Note that building $M$ in this way preserves the adjacencies of $G$, that is $M$ has the same structure as $G$. As stated earlier, no non-trivial simple graph $G$ is totally irregular; it is thus legitimate to wonder how to build a corresponding totally irregular multigraph $M$ in the fashion above. In their work, Chartrand et al. regard edge multiplications as an expensive operation they want to limit as much as possible. This results in the following optimization problem: For a given graph $G$, what is the smallest $k$ such that $G$ can be turned into a totally irregular multigraph $M$ by replacing each edge with at most $k$ parallel edges? From the labelling point of view, this smallest $k$ is precisely $s(G)$.

Since the notion of total irregularity does not fit with simple graphs, as $K_{1}$ is the only totally irregular simple graph, it is legitimate to consider that this notion is too strong, and instead consider weaker notions of irregularity. A few such notions have been explored in the literature, such as the notions of highly irregular graphs [8] or locally irregular graphs [2] we have introduced earlier. With respect to Chartrand et al.'s point of view, we note that there is again a straight connection between proper labellings and locally irregular graphs. Namely, given a proper labelling $\ell$ of a graph $G$, by replacing each edge $e$ with $\ell(e)$ parallel edges, we here get a locally irregular multigraph $M$ with the same structure as $G$. As in the previous problem, one can again ask about the smallest $k$ such that $G$ admits proper $k$-labellings. This smallest $k$ (if any) is denoted $\chi_{\Sigma}(G)$.

This parameter $\chi_{\Sigma}$ is precisely at the heart of maybe the most famous conjecture concerning proper labellings of graphs, the so-called 1-2-3 Conjecture, introduced by Karoński, Łuczak and Thomason in 2004 [12]. This conjecture states that for every nice graph $G$, we have $\chi_{\Sigma}(G) \leq 3$. It is worth noting that there exist nice graphs $G$ that verify $\chi_{\Sigma}(G)=3$ (for example every nice complete graph $K_{n}$ has $\chi_{\Sigma}\left(K_{n}\right)=3$ as shown in [7]) and thus it is meaningless to try to show that $\chi_{\Sigma}(G) \leq 2$ holds for all graphs $G$. There are many results supporting the 1-2-3 Conjecture, the most famous of which belonging to Kalkowski, Karoński and Pfender [11, stating that for any nice graph $G$, we have $\chi_{\Sigma}(G) \leq 5$. Another important result shown in [12] states that the 1-2-3 Conjecture is satisfied for nice 3-colourable graphs. However, unless a graph $G$ is locally irregular (in which case $\chi_{\Sigma}(G)=1$ ), it is not easy in general to characterize which graphs $G$ have $\chi_{\Sigma}(G)=2$ (see [10]). Nevertheless, quite recently a good characterization of nice bipartite graphs $G$ with $\chi_{\Sigma}(G)=3$ was provided in $[18$.

Minimizing, however, the maximum label that is used to create a proper labelling of a graph $G$, does not always guarantee that we have actually minimized the cost that corresponds to the edge multiplications described above. For example, a 2-labelling $\ell$ that assigns label 2 to three edges of $G$ and label 1 to the rest is more expensive (in terms of how many edges the corresponding multigraphs have) than a 3-labelling $\ell^{\prime}$ that assigns label 3 on only one edge of $G$ and label 1 to the rest. The 1-2-3 Conjecture, if true, would imply that every nice graph $G$ admits a proper labelling where the sum of assigned labels is at most $3|E|$; but it might be that, using larger labels, we can design better (with respect to the concerns above) proper labellings of $G$. This motivates us to introduce and study a new problem, namely the problem of finding proper labellings that minimize the sum of the assigned labels.

Formally, for a labelling $\ell$ of a graph $G$, we denote by $\sigma(\ell)$ the sum of labels assigned to the edges of $G$ by $\ell$. That is,

$$
\sigma(\ell):=\sum_{e \in E(G)} \ell(e)
$$

For any $k \geq 1$, we denote by $\operatorname{mE}_{k}(G)$ the minimum value of $\sigma(\ell)$ over all $k$-labellings of $G$. That is,

$$
\operatorname{mE}_{k}(G):=\min \{\sigma(\ell): \ell \text { is a } k \text {-labelling of } G\} \text {. }
$$


We set $\mathrm{mE}(G):=\min \left\{\mathrm{mE}_{k}(G): k \geq \chi_{\Sigma}(G)\right\}$.

The study of the parameter $\operatorname{mE}(G)$ takes place in a recent series of works dedicated to understanding better the connection between proper labellings and proper vertex-colourings of graphs. In [1], the authors studied proper vertex-colourings of graphs that minimize the number of distinct resulting colours. In other words, the authors are there interested in proper labellings by which the number of distinct resulting vertex colours is as close as possible to the chromatic number. Noticing that this minimum number of distinct colours by a proper labelling is bounded above by the minimum maximum vertex colour that can be achieved by a proper labelling, in [5] the authors studied proper labellings where the maximum resulting vertex colour is as small as possible. In these two works, the authors mainly showed that finding an "optimal" proper labelling is hard in general but easy in particular graph classes, and provided results towards conjectures they have raised on their modified notions of proper labellings.

An important point for mentioning the existence of [1] and [5] is that determining $\mathrm{mE}(G)$ for a given graph $G$ is also related to finding a proper labelling of $G$ where the resulting vertex colours satisfy some properties. More precisely, by a straight equivalence between edge labels and vertex colours, see upcoming Observation 2.2 it can be established that determining $\mathrm{mE}(G)$ is equivalent to finding a proper labelling of $G$ that minimizes the sum of resulting vertex colours. Thus, at least at first glance, one could think that determining $\operatorname{mE}(G)$ is somewhat related to the investigations in [1] and [5]. In Section 4.1, we actually show that this is not the case, in the sense that proper labellings that are good for our concerns might be arbitrarily bad for those in [1] and [5], and vice versa.

This work is organized as follows. In Section 2 we provide some useful observations that are going to be used throughout this work. As a warm up, we also provide the exact value of $\operatorname{mE}(G)$ for easy classes of graphs, namely complete bipartite graphs, complete graphs and cycles.

In Section 3 we deal with the algorithmic aspects of the problem. We show that for $k \in \mathbb{N}$, determining $\mathrm{mE}_{k}(G)$ is $\mathcal{N} \mathcal{P}$-complete when $G$ is a planar bipartite graph. Then we provide an algorithm that, given two integers $s$ and $k$, can decide in polynomial time if $\mathrm{mE}_{k}(G) \leq s$ when $G$ belongs to the family of graphs that have bounded treewidth.

In Section 4 we answer two different questions that deal with the particular nature of our problem. First, in Section 4.1 we show that in general a $k$-labelling that minimizes the maximum induced colour does not minimize the sum of the labels used and vice versa. Then, in Section 4.2 we provide an infinite family of graphs $G$ for which $\mathrm{mE}_{k}(G)$ can be arbitrary larger than $\mathrm{mE}_{k+1}(G)$. As mentioned earlier, this property is what justifies the study of this problem, as it shows that just finding a $k$-labelling of $G$ for $k=\chi_{\Sigma}(G)$ is not enough.

Finally, in Section 5 we study the more general aspects of the problem. In particular we propose Conjecture 5.2 stating that for every nice graph $G$, we should have $\operatorname{mE}(G) \leq 2|E(G)|$. We then proceed by providing upper bounds for some families of graphs, namely bipartite graphs and trees, as well as graphs with large chromatic number, that further strengthen our belief that Conjecture 5.2 should hold true.

\section{First observations and classes of graphs}

In this warm-up section, we give some first insight into the problem of determining the parameters $\mathrm{mE}(G)$ and $\mathrm{mE}_{k}(G)$ for a given graph $G$. This is done through first observations on the problem, and by then focusing on easy classes of graphs.

\subsection{First observations and remarks}

The following observation provides trivial bounds on $\sigma(\ell)$ for a $k$-labelling $\ell$ assigning labels with value at most some $k$.

Observation 2.1. Let $G$ be a graph and $\ell$ be a k-labelling of $G$. Then

$$
|E(G)| \leq \sigma(\ell) \leq k|E(G)| .
$$


Consequently, for any $k \geq \chi_{\Sigma}(G)$, we have

$$
|E(G)| \leq \mathrm{mE}_{k}(G) \leq k|E(G)| .
$$

The following provides an obvious way for relating edge labels and vertex colours by a labelling of a graph $G$, that, in general, is a convenient tool for establishing lower bounds on $\mathrm{mE}(G)$.

Observation 2.2. Let $G$ be a graph, and $\ell$ be a labelling of $G$. Then

$$
\sum_{e \in E(G)} 2 \ell(e)=\sum_{v \in V(G)} c_{\ell}(v) .
$$

In particular, by any labelling $\ell$, the sum $\sum_{v \in V(G)} c_{\ell}(v)$ must be an even number.

In several contexts, we will make use of the following property of proper labellings in graphs having some particular sparse structure (adjacent degree-2 vertices):

Observation 2.3. Let $G$ be a graph with a path $\left(v_{1}, v_{2}, v_{3}, v_{4}\right)$ such that $d\left(v_{2}\right)=d\left(v_{3}\right)=2$. Then, by any proper labelling $\ell$ of $G$, we have $\ell\left(v_{1} v_{2}\right) \neq \ell\left(v_{3} v_{4}\right)$.

Proof. This is because $c_{\ell}\left(v_{2}\right)=\ell\left(v_{1} v_{2}\right)+\ell\left(v_{2} v_{3}\right)$ and $c_{\ell}\left(v_{3}\right)=\ell\left(v_{2} v_{3}\right)+\ell\left(v_{3} v_{4}\right)$. This implies we must have $\ell\left(v_{1} v_{2}\right) \neq \ell\left(v_{3} v_{4}\right)$ so that $c_{\ell}\left(v_{2}\right) \neq c_{\ell}\left(v_{3}\right)$.

\section{$2.2 \quad$ Easy classes of graphs}

In this section, we determine what the value of $\operatorname{mE}(G)$ is when $G$ is any nice complete bipartite graph, complete graph, or cycle. Let us recall that, for any nice complete bipartite graph $K_{n, m}$, we have $\chi_{\Sigma}\left(K_{n, m}\right)=1$ if $n \neq m>1$, and $\chi_{\Sigma}\left(K_{n, m}\right)=2$ otherwise. For every nice complete graph $K_{n}$, we have $\chi_{\Sigma}\left(K_{n}\right)=3$. For every nice cycle $C_{n}$, we have $\chi_{\Sigma}\left(C_{n}\right)=3$ whenever $n \geq 3$ is odd or $n \equiv 2 \bmod 4$, while we have $\chi_{\Sigma}\left(C_{n}\right)=2$ otherwise, i.e., when $n \equiv 0 \bmod 4$. Simple proofs for these statements can be found e.g. in [7].

Note that in all the results obtained in this section, while constructing a proper labelling $\ell$ of a graph $G$ achieving $\sigma(\ell)=\operatorname{mE}(G)$, using a label larger than $\chi_{\Sigma}(G)$ is not needed. That is, we here always have $\mathrm{mE}_{k}(G)=\operatorname{mE}(G)$ for $k=\chi_{\Sigma}(G)$. It is important to point out however that this behaviour is not true in general (see Section 4.2).

Theorem 2.4. Let $G=(A, B, E)=K_{n, m}$ be a nice complete bipartite graph with $n+m>2$. Then:

- if $n \neq m$, then $\mathrm{mE}(G)=\mathrm{mE}_{1}(G)=n m=|E(G)|$;

- otherwise, i.e., $n=m$, we have $\mathrm{mE}(G)=\mathrm{mE}_{2}(G)=n(m+1)=|E(G)|+\sqrt{|E(G)|}$.

Proof. If $n \neq m$, then $G$ is locally irregular, in which case we get a 1-labelling when assigning label 1 to all edges. This is best possible due to Observation 2.1. If $n=m, G$ is not locally irregular and thus $k \geq 2$, meaning that a labelling of $G$ must assign a label different from 1 to some edges. Moreover, if a labelling assigns a label different than 1 to less than $n$ edges, then there would necessarily be, in both $A$ and $B$, vertices incident only to edges labelled 1, thus with colour $n$. In that case, $\ell$ would not be proper as some adjacent vertices would have the same colour.

This means that, a proper labelling $\ell$ of $G$ must assign a label different from 1 to at least $n$ edges. This implies that $\mathrm{mE}_{k}(G) \geq|E(G)|+n$. We claim there is a labelling $\ell$ achieving this lower bound, hence best possible. To obtain $\ell$, let $a$ be any vertex of $A$. Assign label 2 to all the $n$ edges incident to $a$, and assign label 1 to all other edges. This labelling is proper. Indeed $c_{\ell}(a)=2 n, c_{\ell}\left(a^{\prime}\right)=n$ for every $a^{\prime} \in A \backslash\{a\}$, and $c_{\ell}(b)=n+1$ for every $b \in B$. Furthermore, $\sigma(\ell)=|E(G)|+n=|E(G)|+\sqrt{|E(G)|}$.

Theorem 2.5. Let $K_{n}$ be a nice complete graph with $n \geq 3$. Then:

- if $n=3$, then $\mathrm{mE}\left(K_{3}\right)=\mathrm{mE}_{3}\left(K_{3}\right)=6=2\left|E\left(K_{3}\right)\right|$; 
- if $n \equiv 0(\bmod 4)$ or $n \equiv 1(\bmod 4)$, then $\mathrm{mE}\left(K_{n}\right)=\mathrm{mE}_{3}\left(K_{n}\right)=\frac{1}{2}\left(n^{2}+\frac{(n-2)(n-1)}{2}-1\right)=$ $\frac{3}{2}\left|E\left(K_{n}\right)\right| ;$

- if $n \equiv 2(\bmod 4)$ or $n \equiv 3(\bmod 4)$, then $\operatorname{mE}\left(K_{n}\right)=\operatorname{mE}_{3}\left(K_{n}\right)=\frac{1}{2}\left(n^{2}+\frac{(n-2)(n-1)}{2}\right)=$ $\left\lceil\frac{3}{2}\left|E\left(K_{n}\right)\right|\right\rceil$.

Proof. Throughout this proof, for any $n \geq 3$, let $V\left(K_{n}\right)=\left\{v_{1}, \ldots, v_{n}\right\}$.

Regarding the first item, Observation 2.3 implies that a proper 3-labelling of $K_{3}$ must assign three distinct labels to the edges, and thus having $\left\{\ell\left(v_{1} v_{2}\right), \ell\left(v_{1} v_{3}\right), \ell\left(v_{2} v_{3}\right)\right\}=\{1,2,3\}$ is optimal, in which case $\sigma(\ell)=6$.

Let us now focus on the second and third items. Following Observation 2.2, finding a proper 3-labelling of $K_{n}$ achieving $\mathrm{mE}_{3}\left(K_{n}\right)$ is equivalent to finding a proper 3-labelling minimizing the sum of vertex colours. Since, in $K_{n}$, all vertices have degree $n-1$, and all vertex colours must be different by a proper 3-labelling, any proper 3-labelling producing distinct vertex colours in $S_{1}=\{n-1, n, n+1, \ldots, 2 n-2\}$ would be optimal. Note, however, that when $n$ is congruent to 2 or 3 modulo 4 , such a proper 3-labelling cannot exist as, in such cases, the sum $n-1+n+(n+$ $1)+\cdots+(2 n-2)$ of the values in $S_{1}$ is odd, which cannot be achieved by a labelling (recall the last statement of Observation 2.2 . In such cases, however, any proper labelling producing distinct vertex colours in $S_{2}=\{n-1, n, n+1, \ldots, 2 n-3,2 n-1\}$ would be optimal.

Now consider the following 3-labelling $\ell$ of $K_{n}(n \geq 4)$, already introduced in [5], to establish what the value $\mathrm{mS}_{3}\left(K_{n}\right)$ is. We label the edges of $K_{n}$ through three steps. Firstly, we assign label 1 to every edge. Secondly, we change the labels of the edges in $\left\{v_{i} v_{j} \mid 1 \leq i, j \leq n, i+j \geq n+2\right\}$ to 2 . Then $v_{1}$ is incident to no edge labelled 2 , vertex $v_{2}$ is incident to one edge labelled 2 , vertex $v_{i}$ for $3 \leq i \leq\lfloor(n-1) / 2\rfloor+1$ is incident to $i-1$ edges labelled 2 , and $v_{i}$ for $\lfloor(n-1) / 2\rfloor+2 \leq i \leq n$ is incident to $i-2$ edges labelled 2. Let $j=\lfloor(n-1) / 2\rfloor+1$. Note that for every $i \in\{2,3, \ldots, j, j+2, \ldots, n\}, v_{i}$ is adjacent to one more edge labelled 2 than $v_{i-1}$; and that $v_{j}$ and $v_{j+1}$ are both adjacent to $j-1$ edges labelled 2 (and $n-j$ edges labelled 1). So $c_{l}\left(v_{1}\right)<c_{l}\left(v_{2}\right)<\cdots<c_{l}\left(v_{j}\right)=c_{l}\left(v_{j+1}\right)<c_{l}\left(v_{j+2}\right)<$ $\cdots<c_{l}\left(v_{n}\right)$ and $c_{l}\left(v_{i+1}\right) \leq c_{l}\left(v_{i}\right)+1$ for $1 \leq i \leq n$, i.e., all vertices have different colours except $v_{j}$ and $v_{j+1}$. Finally, to avoid the conflict between $v_{j}$ and $v_{j+1}$, let us increase the label of $v_{j+1} v_{j+2}$ from 2 to 3 . This change induces a new conflict between $v_{j+2}$ and $v_{j+3}$. Then we need to increase the label of $v_{j+3} v_{j+4}$ from 2 to 3 to get rid of this conflict, which creates a new conflict, and so on. Formally, we change the labels of the edges in $\left\{v_{j+1} v_{j+2}, v_{j+3} v_{j+4}, \ldots, v_{n-1} v_{n}\right\}$ to 3 if $n-j$ is even, i.e., if $n \equiv 0 \bmod 4$ or $n \equiv 1 \bmod 4$. Otherwise, if $n-j$ is odd and $n \equiv 2 \bmod 4 \operatorname{or} n \equiv 3 \bmod 4$, then we change the labels of the edges in $\left\{v_{j+1} v_{j+2}, v_{j+3} v_{j+4}, \ldots, v_{n-4} v_{n-3}, v_{n-2} v_{n}, v_{n-1} v_{n}\right\}$ to 3 .

It can be checked that the resulting 3-labelling $\ell$ is proper, and achieves vertex colours in $S_{1}$ when $n$ is congruent to 0 or 1 modulo 4 , or vertex colours in $S_{2}$ when $n$ is congruent to 2 or 3 modulo 4. As discussed above, this is best possible. Furthermore, it can easily be checked that the elements in $S_{1}$ sum up to the value claimed in the second item, and similarly for the elements in $S_{2}$ and the value claimed in the third item. This concludes the proof.

Theorem 2.6. Let $n \geq 3$, and $C_{n}$ be the nice cycle of length $n$. Then:

- if $n \equiv 0(\bmod 4)$, then $\mathrm{mE}\left(C_{n}\right)=\mathrm{mE}_{2}\left(C_{n}\right)=\frac{3}{2}\left|E\left(C_{n}\right)\right|$;

- if $n \equiv 1(\bmod 4)$ or $n \equiv 3(\bmod 4)$, then $\left.\operatorname{mE}\left(C_{n}\right)=\operatorname{mE}_{3}\left(C_{n}\right)=\left\lceil\frac{3}{2} \mid E\left(C_{n}\right)\right\rceil\right\rceil+1$;

- if $n \equiv 2(\bmod 4)$, then $\operatorname{mE}\left(C_{n}\right)=\mathrm{mE}_{3}\left(C_{n}\right)=\frac{3}{2}\left|E\left(C_{n}\right)\right|+3$.

Proof. Let us order the edges of $C_{n}$ following a clockwise direction and define $E\left(C_{n}\right)=\left\{e_{1}, \ldots, e_{n}\right\}$ and $V\left(C_{n}\right)=\left\{v_{1}, \ldots, v_{n}\right\}$ such that for $i<n, e_{i}=v_{i} v_{i+1}$ and $e_{n}=v_{n} v_{1}$. Thus for $i>0$, $N\left(v_{i}\right)=\left\{v_{i-1}, v_{i+1}\right\}$ and $N\left(v_{1}\right)=\left\{v_{2}, v_{n}\right\}$. Recall that $\chi_{\Sigma}\left(C_{n}\right)=2$ for the first item and that $\chi_{\Sigma}\left(C_{n}\right)=3$ for the second and third item.

Claim 2.7. Let $l \leq k$ and $\ell$ be a $k$-labelling of $C_{n}$ that assigns label $l$ to at least one edge. If $\ell$ is proper, then it assigns label $l$ to at most $\left\lfloor\frac{1}{2}\left|E\left(C_{n}\right)\right|\right\rfloor$ edges if $n$ is odd and to at most $\frac{1}{2}\left|E\left(C_{n}\right)\right|-1$ edges if $n \equiv 2(\bmod 4)$. 
Proof of the claim. Let $\mathcal{E}=\left\{e \in E\left(C_{n}\right): \ell(e)=l\right\}$ and $G=(V, E)$ be the graph that has $V=\left\{v_{i}: e_{i} \in E\left(C_{n}\right)\right\}$ and for $i \neq j, v_{i} v_{j} \in E$ if the corresponding edges $e_{i}, e_{j}$ are at distance exactly 2 in $C_{n}$. Obviously $\left|E\left(C_{n}\right)\right|=|V|$. It follows from Observation 2.3 that if $\ell$ is a proper labelling for $C_{n}$ that maximizes $|\mathcal{E}|$, then $|\mathcal{E}|=|S|$, where $S$ is an independent set of $G$. For $n$ odd, $G=C_{n}$. Since $G$ is a cycle, $|\mathcal{E}|=|S|=\left\lfloor\frac{1}{2}|V|\right\rfloor=\left\lfloor\frac{1}{2}\left|E\left(C_{n}\right)\right|\right\rfloor$. For $n \equiv 2(\bmod 4)$, let $m$ be such that $n=4 m+2$. It is clear that $G$ contains two connected components, each one being a copy of the $C_{\frac{n}{2}}$ cycle. Thus, $|\mathcal{E}|=|S|=2\left\lfloor\frac{n}{2}\right\rfloor=2 m=\frac{1}{2}\left|E\left(C_{n}\right)\right|-1$.

For the first item let $\ell$ be the following 2-labelling: $\ell\left(e_{1}\right)=1, \ell\left(e_{2}\right)=1, \ell\left(e_{3}\right)=2, \ell\left(e_{4}\right)=$ $2, \ell\left(e_{5}\right)=1, \ldots, \ell\left(e_{n}\right)=2$. Let us assume that this $\ell$ is not proper. Then there would exist at least two adjacent vertices $v_{i}, v_{i+1}$ such that $c_{\ell}\left(v_{i}\right)=c_{\ell}\left(v_{i+1}\right)$. It follows that $\ell\left(v_{i-1}\right)=\ell\left(v_{i+2}\right)$ (if $i=1$ then $v_{i-1}=v_{n}$ and if $i=n$ then $\left.v_{i+1}=v_{1}\right)$ which is a contradiction. Furthermore, since $n \equiv 0$ $(\bmod 4)$, label 2 is used on exactly half the edges of $C_{n}$ and thus $\sigma(\ell)=\left|E\left(C_{n}\right)\right|+\frac{1}{2}\left|E\left(C_{n}\right)\right|=$ $\frac{3}{2}\left|E\left(C_{n}\right)\right|$. Moreover, this value is optimal. Indeed, assume it is not. Then, there would exist a labelling $\ell^{\prime}$ such that more than $\frac{1}{2}\left|E\left(C_{n}\right)\right|$ edges are labelled 1 by $\ell^{\prime}$, a contradiction by Claim 2.7

Let $C_{n}$ be a cycle with $n \equiv 1(\bmod 4)$. We will show that $\operatorname{mE}\left(C_{n}\right)=\left\lceil\frac{3}{2}\left|E\left(C_{n}\right)\right|\right\rceil+1$. Let $\ell$ be a labelling of $C_{n}$ that assigns label 3 on only one edge. It follows from Claim 2.7 that at most $\left\lfloor\frac{n}{2}\right\rfloor$ edges of $C_{n}$ are labelled 1. Actually, there are exactly $\left\lfloor\frac{n}{2}\right\rfloor$ edges labelled 1: if this was not the case, and since only one edge of $C_{n}$ is labelled 3, there would be more than $\left\lfloor\frac{n}{2}\right\rfloor$ edges labelled 2, which contradicts Claim 2.7. The same holds true for the edges labelled 2. Since $n \equiv 1(\bmod 4) \Rightarrow \exists m$ : $n=4 m+1$ and using this, one can easily show that $\sigma(\ell)=\left\lfloor\frac{n}{2}\right\rfloor+2\left\lfloor\frac{n}{2}\right\rfloor+3=\cdots=\left\lceil\frac{3}{2} \mid E\left(C_{n}\right)\right\rceil+1$. Furthermore, let $\ell^{\prime}$ be a labelling of $C_{n}$ that assigns label 3 on more than one edge. It is clear that if $\ell^{\prime}$ is proper, then $\sigma(\ell)<\sigma\left(\ell^{\prime}\right)$. Thus, $\sigma(\ell)=\mathrm{mE}\left(C_{n}\right)$. The following is a proper labelling that achieves this optimal value: $\ell\left(e_{1}\right)=1, \ell\left(e_{2}\right)=1, \ell\left(e_{3}\right)=2, \ell\left(e_{4}\right)=2, \ell\left(e_{5}\right)=1, \ldots, \ell\left(e_{n-1}\right)=$ $2, \ell\left(e_{n}\right)=3$.

Let $C_{n}$ be a cycle with $n \equiv 3(\bmod 4)$. Similarly to before, $\operatorname{mE}\left(C_{n}\right)=\left\lceil\frac{3}{2}\left|E\left(C_{n}\right)\right|\right\rceil+1$. The following is a proper labelling that achieves this optimal value: $\ell\left(e_{1}\right)=1, \ell\left(e_{2}\right)=1, \ell\left(e_{3}\right)=$ $2, \ell\left(e_{4}\right)=2, \ell\left(e_{5}\right)=1, \ldots, \ell\left(e_{n-3}\right)=2, \ell\left(e_{n-2}\right)=1, \ell\left(e_{n-1}\right)=3, \ell\left(e_{n}\right)=2$.

Let $C_{n}$ be a cycle with $n \equiv 2(\bmod 4)$. We will show that $\operatorname{mE}\left(C_{n}\right)=\frac{3}{2}\left|E\left(C_{n}\right)\right|+3$. Indeed, let $\ell$ be a labelling of $C_{n}$ that assigns label 3 on only one edge. If $\ell$ is proper, it is obliged to assign label 1 to at most $\frac{1}{2}\left|E\left(C_{n}\right)\right|-1$ edges of $C_{n}$ and label 2 to the rest. This however would lead to $\ell$ assigning label 2 on at least $\frac{1}{2}\left|E\left(C_{n}\right)\right|$ edges, which is a contradiction by Claim 2.7. Thus, $\ell$ must assign label 3 on at least two edges. Similarly to before, a labelling that assigns label 3 on exactly two, label 1 to at most $\frac{1}{2}\left|E\left(C_{n}\right)\right|-1$ edges and label 2 to the rest, would achieve the optimal value. The following is one such proper labelling: $\ell\left(e_{1}\right)=1, \ell\left(e_{2}\right)=1, \ell\left(e_{3}\right)=2, \ell\left(e_{4}\right)=2, \ell\left(e_{5}\right)=$ $1, \ldots, \ell\left(e_{n-3}\right)=2, \ell\left(e_{n-2}\right)=2, \ell\left(e_{n-1}\right)=3, \ell\left(e_{n}\right)=3$.

\section{Complexity aspects}

In this section, we establish both a negative and a positive result on the complexity of computing the parameter $\mathrm{mE}_{k}(G)$ for some input integer $k$ and nice graph $G$. More precisely:

- We first prove that determining $\mathrm{mE}_{2}(G)$ is $\mathcal{N} \mathcal{P}$-complete, even when $G$ is restricted to a planar bipartite graph. Recall that this is contrasting with the complexity of determining whether $\chi_{\Sigma}(G) \leq 2$ holds for a given bipartite graph $G$, which is a problem that can be solved in polynomial time due to a result of Thomassen, Wu and Zhang [18].

- We then prove that determining $\mathrm{mE}_{k}(G)$ can be done in polynomial time whenever $k$ is fixed and $G$ is a graph with bounded treewidth.

\subsection{A negative result for bipartite graphs}

In this section, we prove, in Theorem 3.2 below, that the problem of determining $\mathrm{mE}_{k}(G)$ is $\mathcal{N P}$-complete in planar bipartite graphs $G$.

Let us first introduce the $k$-gadget, for $k \geq 11$, which will be useful for proving Theorem 3.2 . To build this gadget, let us start with $k-1$ stars, each having a center denoted by $s_{i}, i \in[1, k-1]$, 
such that $d\left(s_{i}\right)=k+1$. For each star, we pick an arbitrary edge $s_{i} y_{i}$ and identify all the $y_{i}$ into a single vertex $y$, which we call the representative of the gadget. Finally we add another vertex $u$, called the root of the gadget, which is connected to $y$. It is clear that $d(u)=1$ and $d(y)=k$. Also note that each $k$-gadget is a tree with $\mathcal{O}\left(k^{2}\right)$ edges.

When we say that we attach a gadget $H$ to the vertex $v$ of a graph $G$ we mean that we add $H$ to $G$ and the root $u$ of the gadget is identified to $v$.

Claim 3.1. Let $G(V, C, E)$ be a bipartite graph and $\ell$ be any 2-labelling of $G$ such that $\sigma(\ell) \leq$ $|E(G)|+c$, for $c=|C|$. Let $H$ be any p-gadget attached to $G$, where $p-1>c$. Let $y$ be the representative of $H$. If at least one edge $e$ of $H$ incident to $y$ is labelled 2 , then there are at least two edges of $H$ that are labelled 2.

Proof of the claim. Let us suppose that at least one of the edges of $H$ incident to $y$ is labelled 2. Let $z \in V \cup C$ be the vertex of $G^{\prime}$ to which $H$ has been attached.

Let us first assume that the edge $z y$ is labelled 2. If $y$ is incident to only a single edge labelled 2 (i.e., $z y$ ), then its colour is $c_{\ell}(y)=p+1$. Since all its $p-1$ neighbours $(\neq z)$ have degree $p+1$, each of them must be incident to at least one edge labelled 2 as otherwise they would have the same colour as $y$. This leads to at least $p>c$ edges labelled 2, which is a contradiction (observe that since $\sigma(\ell) \leq|E(G)|+c$, there are at most $c$ edges labelled 2). Otherwise, if $y$ has exactly one other incident edge $(\neq y z)$ labelled 2 , say the edge $y w$, and we are done.

Then, let us assume that $\ell(y z)=1$. Moreover, let us assume that some edge incident to $y$, say $y w_{1}$ different than $y z$, is labelled 2. Then $c_{\ell}(y) \geq p+1$ with $c_{\ell}(y)=p+1$ if $y w_{1}$ is the unique edge incident to $y$ labelled 2. In this case, each one of the $p-2$ neighbours of $y\left(\neq z, w_{1}\right)$ must be incident to at least one edge labelled 2 , leading to at least $p-1>c$ edges labelled 2 , which is a contradiction. Thus $c_{\ell}(y)>p+1$, which means there is at least one more edge $y w_{2}$ incident to $y$ labelled 2.

We are now ready for proving our result.

Theorem 3.2. Let $G$ be a nice planar bipartite graph, $k \geq 2$ and $q \in \mathbb{N}$. The problem of deciding if $\mathrm{mE}_{k}(G) \leq q$ is $\mathcal{N} \mathcal{P}$-complete.

Proof. The problem is clearly in $\mathcal{N} \mathcal{P}$. We focus on showing it is also $\mathcal{N} \mathcal{P}$-hard. The proof is done by reduction from Planar Monotone 1-IN-3 SAT, which was shown to be $\mathcal{N} \mathcal{P}$-complete in [14]. In this problem, a $3 \mathrm{CNF}$ formula $F$ is given as input, which has clauses with exactly three variables all of which appear only positively. We say that a bipartite graph $G^{\prime}=(V, C, E)$ corresponds to $F$ if it is constructed in the following way: for each variable $x_{i}$ of $F$ we add a variable vertex $v_{i}$ in $V$ and for each clause $C_{j}$ of $F$ we add a clause vertex $c_{j}$ in $C$. Then the edge $v_{i} c_{j}$ is added if variable $x_{i}$ appears in clause $C_{j}$. Furthermore, $F$ is valid as input to the Planar Monotone 1-IN-3 SAT problem if the graph $G^{\prime}$ that corresponds to $F$ is planar. The question is, whether there exists a 1-in-3 truth assignment of $F$; that is a truth assignment to the variables of $F$ such that each clause has exactly one variable with the value true.

Let us prove the statement for $k=2$. Let $F$ be the 3CNF formula, with $c$ clauses that is given as input to the Planar Monotone 1-IN-3 SAT problem. Our goal is to construct a planar bipartite graph $G$ such that $F$ is 1-in-3 satisfied if and only if $\mathrm{mE}_{2}(G) \leq|E(G)|+c$.

We start with $G^{\prime}=(V, C, E)$ being the planar bipartite graph that corresponds to $F$, with $V$ being the set of the variable vertices $v_{i}$ and $C$ being the set of the clause vertices $c_{j}$. Note that in $F$, each clause has exactly three variables but there is no bound on how many times a variable appears in $F$. Thus for each $v_{i} \in V, d\left(v_{i}\right) \geq 1$ and for each $c_{j} \in C, d\left(c_{j}\right)=3$. It follows that $|C|=\mathrm{c}$ and $|V| \leq 3 c$.

We proceed by modifying $G^{\prime}$ by adding the $k$-gadgets we described earlier. For each variable vertex $v_{i}$ of $G$, let $d_{i}$ be the degree of $v_{i}$ in $G^{\prime}$. Let $d_{v, i}=\left(d_{i}-1\right)(c+1)+d_{i}$ and $d_{c}=3(c+1)+3$. For each variable vertex $v_{i}$, for all $1 \leq j<d_{i}$, we attach $c+1$ copies of the $\left(d_{v, i}+j\right)$-gadget. Thus the degree of each $v_{i}$ in $G$ becomes equal to $d_{v, i}$. On each clause vertex $c_{j}$, let us attach $c+1$ copies of the $d_{c}$-gadget, $c+1$ copies of the $\left(d_{c}+2\right)$-gadget and $c+1$ copies of the $\left(d_{c}+3\right)$-gadget. Thus the degree of each $c_{j}$ in $G$ becomes equal to $d_{c}$. Clearly, the construction of $G$ is achieved in polynomial time. Finally observe that since $G^{\prime}$ is planar and the attached gadgets are actually trees, $G$ is also planar. 
Let $\ell$ be a 2-labelling of $G$ such that $\sigma(\ell) \leq|E(G)|+c$, i.e., there are at most $c$ edges of $G$ labelled 2 by $\ell$. Observe that $G$ contains $p$-gadgets for $p \in\left\{d_{v, i}+1, d_{v, i}+2, \ldots d_{v, i}+d_{i}-1, d_{c}, d_{c}+1, d_{c}+3\right\}$ and $d_{v, i}-1, d_{c}-1>c$. Thus Claim 3.1 holds for each gadget attached to $G$.

Claim 3.3. For any 2-labelling $\ell$ of $G$ such that $\sigma(\ell) \leq|E(G)|+c$, we have that:

- for each variable vertex $v_{i} \in V, c_{\ell}\left(v_{i}\right) \notin\left\{d_{v, i}+1, d_{v, i}+2, \ldots, d_{v, i}+d_{i}-1\right\}$

- for each clause vertex $c_{j} \in C, c_{\ell}\left(c_{j}\right) \notin\left\{d_{c}, d_{c}+2, d_{c}+3\right\}$

Proof of the claim. Indeed, each variable vertex $v_{i}$ is adjacent to $c+1$ copies of the $\left(d_{v, i}+1\right)$-gadget and at most $c$ edges are labelled 2 by $\ell$. Thus, at least one of the $\left(d_{v, i}+1\right)$-gadgets, let us call it $H$, that is attached to $v_{i}$, has all of its edges labelled 1 . Moreover, $v_{i}$ is adjacent to the representative $y$ of $H$ which has degree $d(y)=d_{v, i}+1$. Since all the edges of $H$ are labelled 1 , the colour $c_{\ell}(y)$ of $y$ is $d_{v, i}+1$ and thus this colour is forbidden for $v_{i}$, i.e., $c_{\ell}\left(v_{i}\right) \neq d_{v, i}+1$.

By repeating the same argument for the $\left(d_{v, i}+2\right)$-gadgets attached to $v_{i}, c_{\ell}\left(v_{i}\right) \neq d_{v, i}+2$. Similarly, by considering the $d_{c}$-gadgets (resp., the $\left(d_{c}+2\right)$ - and $\left(d_{c}+3\right)$-gadgets) attached to any clause vertex $c_{j}, c_{\ell}\left(c_{j}\right) \notin\left\{d_{c}, d_{c}+2, d_{c}+3\right\}$.

Claim 3.4. Let $\ell$ be any 2-labelling of $G$ such that $\sigma(\ell) \leq|E(G)|+c$. Then all edges of the attached gadgets must be labelled 1 .

Proof of the claim. Observe that for each clause vertex $z \in C$, at least one of its incident edges must be labelled 2. If this were not the case, then $c_{\ell}(z)=d_{c}$, and this is not allowed from Claim 3.3 .

Let $H$ be a $k$-gadget attached to $z$ and $y$ be the representative of $H$. Suppose $\ell(y z)=2$. It follows from Claim 3.1 that there are at least two edges of $H$ labelled 2. Recall that the number of edges of $G$ that can be labelled 2 is at most $c$. Thus, the number of edges of $G$, that do not belong to $H$ and can be labelled 2 , is at most $c-2$. Furthermore, there are $c-1$ clause vertices in $G$ that are different from $z$. It follows that there exists a clause vertex that has all of its incident edges labelled 1, a contradiction. Thus, each $z \in C$ must be incident to an edge $w z$ with $\ell(w z)=2$ and $w$ cannot belong to a gadget attached to $w$. It follows that there must be $|C|=c$ edges of $G^{\prime}$ labelled 2 and since $\sigma(\ell) \leq|E(G)|+c$, all the edges of the attached gadgets are labelled 1 .

It follows from Claim 3.4 , that the only possible colours induced by $\ell$ on the vertices of $G^{\prime}$ are:

- for each variable vertex $v_{i} \in V, c_{\ell}\left(v_{i}\right) \in\left\{d_{v, i}, d_{v, i}+1, d_{v, i}+2, \ldots, d_{v, i}+d_{i}-1, d_{v, i}+d_{i}\right\}$

- for each clause vertex $c_{j} \in C, c_{\ell}\left(c_{j}\right) \in\left\{d_{c}, d_{c}+1, d_{c}+2, d_{c}+3\right\}$

Using Claim 3.3 it follows that

- for every variable vertex $v_{i}, c_{\ell}\left(v_{i}\right) \in\left\{d_{v, i}, d_{v, i}+d_{i}\right\}$. Observe that $c_{\ell}\left(v_{i}\right)=d_{v, i}$ if all edges of $G^{\prime}$ adjacent to $v_{i}$ are labelled 1 and $c_{\ell} v_{i}=d_{v, i}+d_{i}$, if all edges of $G^{\prime}$ adjacent to $v_{i}$ are labelled 2.

- for every clause vertex $c_{j}, c_{\ell}\left(c_{j}\right)=\left\{d_{c}+1\right\}$, which corresponds to two edges of $G^{\prime}$ adjacent to $c_{j}$ labelled 1 and only one edge labelled 2 .

We are now ready to show the equivalence between finding a 1-in-3 truth assignment $\phi$ of $F$ and finding a 2-labelling $\ell$ of $G$ such that $\sigma(\ell)=\mathrm{mE}_{2}(G) \leq|E(G)|+c$. An edge $v_{i} c_{j}$ of $G^{\prime}$ labelled 2 (respectively 1 ) by $\ell$ corresponds to variable $x_{i}$ bringing truth value true (respectively false) to clause $C_{j}$ by $\phi$. Also, we know that in $G^{\prime}$, each variable vertex $v_{i}$ is adjacent to $n \geq 1$ edges, all having the same label (either 1 or 2). Accordingly, the corresponding variable $x_{i}$ brings, by $\phi$, the same truth value to the $n$ clauses of $F$ that contain it. Finally, in $G^{\prime}$, each clause vertex $c_{j}$ is adjacent to two edges labelled 1 and one labelled 2 . This corresponds to the clause $C_{j}$ being regarded as satisfied by $\phi$ only when it has exactly one true variable. 


\subsection{A positive result for graphs with bounded treewidth}

Given a graph $G=(V, E)$, a tree-decomposition [] of $G$ is any pair $(T, \mathcal{X})$ such that $T=$ $(V(T), E(T))$ is a tree and $\mathcal{X}=\left\{X_{t} \subseteq V \mid t \in V(T)\right\}$ is a familly of subsets (called bags) of vertices of $G$ such that: $V=\bigcup_{t \in V(T)} X_{t}$; for every $\{u, v\} \in E$, there exists $t \in V(T)$ with $u, v \in X_{t}$; and for every $v \in V,\left\{t \in V(T) \mid v \in X_{t}\right\}$ induces a subtree of $T$. The width of $(T, \mathcal{X})$ is equal to $\max _{t \in V(T)}\left|X_{t}\right|-1$ and the tree-width $t w(G)$ is the minimum width of a tree-decomposition of $G$.

A tree-decomposition $(T, \mathcal{X})$ is nice [6] if $T$ is rooted in $r \in V(T)$ and every node $t \in V(T)$ is exactly one of the following four types:

1. Leaf: $t$ is a leaf of $T$ and $\left|X_{t}\right|=1$

2. Introduce: $t$ has a unique child $t^{\prime}$ and there exists $v \in V$ s.t. $X_{t}=X_{t^{\prime}} \cup\{v\}$

3. Forget: $t$ has a unique child $t^{\prime}$ and there exists $v \in V$ s.t. $X_{t^{\prime}}=X_{t} \cup\{v\}$

4. Join: $t$ has exactly two children $t^{\prime}, t^{\prime \prime}$ and $X_{t}=X_{t^{\prime}}=X_{t^{\prime \prime}}$

Let $G=(V, E)$ be a graph. It is well known that $G$ admits a nice tree-decomposition $(T, \mathcal{X})$ rooted in $r \in V(T)$, that has width equal to $t w(G),|V(T)|=\mathcal{O}(|V|)$ and $X_{r}=\{\emptyset\}[6]$.

Let $(T, \mathcal{X})$ be a rooted tree-decomposition (with root $r$ ) of $G$ and $t \in V(T)$. A subtree of $T$ induced by $t$ and its descendants is denoted as $T_{t}$ and the corresponding subgraph of $G$, i.e. the graph induced by $\cup_{t^{\prime} \in V\left(T_{t}\right)} X_{t^{\prime}}$, is denoted by $G_{t}$ (clearly, $G_{r}=G$ ). For every $v \in V\left(G_{t}\right)$, let $N_{t}(v)$ denote the neighborhood of $v$ in $G_{t}$, that is $N_{t}(v)=\left\{u \in V\left(G_{t}\right): u v \in E\left(G_{t}\right)\right\}$. A quasi k-labelling of $G_{t}$ consists of a pair of functions $(\ell, c)$, with $\ell: E\left(G_{t}\right) \rightarrow\{1, \ldots, k\}$ and $c: V\left(G_{t}\right) \rightarrow \mathbb{N}$, such that $c$ is a proper colouring of $G_{t}$, for every $v \in V\left(G_{t}\right) \backslash X_{t}$ we have $c(v)=c_{\ell}(v)$ and for every $v \in V\left(X_{t}\right), c(v) \geq c_{\ell}(v)$. Intuitively, the notion of quasi $k$-labelling is a generalisation of $k$-labelling that allows us to further modify the labels (and thus the induced colours) of the edges of $X_{t}$ if this is needed in order to extend a $k$-labelling of $G_{t}$ into a $k$-labelling of $G_{t^{\prime}}$, where $t^{\prime}$ is the parent of $t$ in $T$. Finally, let $s_{t}(\ell)=\sum_{e \in E\left(G_{t}\right)} \ell(e)$.

Observe that every $k$-labelling $\ell^{\prime}$ of $G$ induces a quasi $k$-labelling of $G_{t}$. For every $e \in E\left(G_{t}\right)$ and $v \in V\left(G_{t}\right)$, let $\ell(e)=\ell^{\prime}(e)$ and $c(v)=c_{\ell^{\prime}}(v)$. The pair $(\ell, c)$ is a quasi $k$-labelling of $G_{t}$. Indeed, since $(T, \mathcal{X})$ is a tree-decomposition of $G$, for every internal node $t$ of $T, X_{t}$ is a separator between $G_{t}-X_{t}$ and $G-V\left(G_{t}\right)$. Put differently, there are no edges between vertices of $G_{t}-X_{t}$ and $G-V\left(G_{t}\right)$. Furthermore, if $r$ is such that $X_{r}=\emptyset$, then a quasi $k$-labelling of $G_{r}$ is a $k$-labelling of $G$. Indeed, it is true (by definition) that a quasi $k$-labelling of $G_{r}$ differs from a $k$-labelling only on the vertices of $X_{r}$ and since $X_{r}=\emptyset$ and $G_{r}=G$ the observation follows.

Theorem 3.5. Let $k \geq 2$ and tw $\geq 1$ be two fixed integers. Given a nice graph $G=(V, E)$ with $|V|=n$ and an integer $s$, the problem of deciding whether $\mathrm{mE}_{k}(G) \leq s$ can be decided in polynomial time if $G$ belongs to the family of graphs that have width at most tw (and in linear time if $G$ is additionally of bounded maximum degree).

Proof. Let us start by defining some sets. For every $t \in V(T)$, let $\left|X_{t}\right|=w_{t},\left|E\left(G\left[X_{t}\right]\right)\right|=q_{t}$, $X_{t}=\left\{v_{1}, \cdots, v_{w_{t}}\right\}$ and $E\left(G\left[X_{t}\right]\right)=\left\{e_{1}, \cdots, e_{q_{t}}\right\}$ (to simplify the notation, we will simply denote $q_{t}$ and $w_{t}$ by $q$ and $w$ respectively). Let $\mathcal{F}_{t}=\{1, \ldots, k\}^{q} \times\{1, \ldots, k \Delta\}^{w} \times\{0, \ldots, k \Delta\}^{w}$ and $(L, F C, C B) \in \mathcal{F}_{t}$, where $L=\left\{l_{1}, \ldots, l_{q}\right\}, F C=\left\{f_{1}, \ldots, f_{w}\right\}$ and $C B=\left\{b_{1}, \ldots, b_{w}\right\}$. The labels we "intent" to assign to the edges of $X_{t}$ are in $L$, the "final colours" induced by these labels on the vertices of $X_{t}$ are in $F C$ and in $C B$ we can find the contribution to these colours that come "from bellow" (meaning the part of these final colours that are due to edges between $X_{t}$ and $G_{t}-X_{t}$ ). Furthermore, for $X_{t^{\prime}} \subseteq X_{t}$ with $X_{t^{\prime}}=\left\{u_{1}, \ldots, u_{w^{\prime}}\right\}$ (where $w^{\prime} \leq w$ ), let $\left.F C\right|_{X_{t^{\prime}}}=\left\{f_{1}^{\prime}, \ldots, f_{w}^{\prime}\right\}$ be defined by setting $f_{j}^{\prime}=f_{j}$ for each $j \in\left[1, w^{\prime}\right]\left(\left.L\right|_{X_{t^{\prime}}}\right.$ and $\left.C B\right|_{X_{t^{\prime}}}$ are defined similarly).

Precisely, a quasi labelling $(\ell, c)$ of $G_{t}$ is said compatible with $(L, F C, C B) \in \mathcal{F}_{t}$ if, for each $i \in[1, q]$ and $j \in[1, w]$, we have that $\ell\left(e_{i}\right)=l_{i}, c\left(v_{j}\right)=f_{j}$, and $b_{j}=\sum_{x \in N_{t}\left(v_{j}\right) \backslash X_{t}} \ell\left(v_{j} x\right)$. Note that this implies that, for all $i \in[1, q]$ and $j \in[1, w]$,

$$
\sum_{z \in N_{t}\left(v_{j}\right)} \ell\left(z v_{j}\right)=b_{j}+\sum_{z \in N\left(v_{j}\right) \cap X_{t}, e_{i}=v_{j} z} l_{i} \leq c\left(v_{j}\right)=f_{j}
$$


By $\left(\ell^{*}, c^{*}\right)$ we denote one such compatible labelling that minimizes the sum of the labels assigned by $\ell^{*}$ among all quasi-labellings compatible with $(L, F C, C B)$. That is, for any $t \in V(t), s_{t}\left(\ell^{*}\right) \leq$ $s_{t}(\ell)$ for every quasi-labelling $\ell$ compatible with $(L, F C, C B)$. Let $\alpha_{t}(L, F C, C B):=s_{t}\left(\ell^{*}\right)$. In essence, for each possible $(L, F C, C B) \in \mathcal{F}_{t}, \alpha_{t}(L, F C, C B)$ is equal to the sum of the labels of an optimal (in terms of sum of labels) quasi labelling $\left(\ell^{*}, c^{*}\right)$ of $G_{t}$ that is compatible with $(L, F C, C B)$. Note that not all $(L, F C, C B) \in \mathcal{F}_{t}$ admit compatible quasi-labelling. If $(L, F C, C B) \in \mathcal{F}_{t}$ has no compatible quasi-labelling, we set $\alpha_{t}(L, F C, C B)=\infty$.

Finally, let us set

$$
\operatorname{Table}(t)=\left(\left(L, F C, C B, \alpha_{t}(L, F C, C B)\right)\right)_{(L, F C, C B) \in \mathcal{F}_{t}}
$$

being the table associated to each $t \in V(T)$. Note that $\mid$ Table $(t) \mid=\mathcal{O}\left(k^{q}(k \Delta+1)^{2 w}\right)=$ $\mathcal{O}\left(k^{(t w(G)+1)^{2}}(k \Delta+1)^{2 t w(G)+2}\right)$, since $q \leq\left(\begin{array}{c}t w(G)+1 \\ 2\end{array}\right)=\mathcal{O}\left((t w(G)+1)^{2}\right)$ and $w \leq t w(G)+1$. Furthermore, since $r$ is such that $X_{r}=\emptyset, \alpha_{r}=\alpha_{r}(\emptyset, \emptyset, \emptyset)$ is equal to the sum of an optimal $k$-labelling of $G_{r}$ and thus Table $(r)=\left(\left(\emptyset, \emptyset, \emptyset, \alpha_{r}\right)\right)$, where $\alpha_{r}=\mathrm{mE}_{k}(G)$. All that remains to be done is to compute this Table $(t)$ for every $t \in V(t)$. We present a dynamic programming algorithm that performs this computation bottoms up; that is starting from the leaves of $T$ and progressing towards $r$. The computation depends on the type of $t$.

Let $t$ be a leaf node. Recall that $\left|X_{t}\right|=1$ and thus there are no edges in $X_{t}$. For every $y \in[1, \cdots k \Delta]$ and every $(L, F C, C B) \in \mathcal{F}_{t}$, the $\alpha_{t}(L, F C, C B)$ entry of Table $(t)$ is defined as:

$$
\alpha_{t}(L, F C, C B)= \begin{cases}0, & \text { if }(L, F C, C B)=(\emptyset,\{y\},\{0\}) \\ \infty, & \text { otherwise }\end{cases}
$$

Let $t$ be an introduce node, $t^{\prime}$ be its unique child, let $w=\left|X_{t^{\prime}}\right|$, and let $v$ be such that $X_{t}=X_{t^{\prime}} \cup\{v\}=\left\{v_{1}, \cdots, v_{w}, v_{w+1}=v\right\}$. Moreover, let $E\left(X_{t}\right)=\left\{e_{1}, \ldots, e_{q}, e_{q+1}, \ldots, e_{q+h}\right\}$, where $E\left(X_{t^{\prime}}\right)=\left\{e_{1}, \ldots, e_{q}\right\}$. Essentially, the set $\left\{e_{q+1}, \ldots, e_{q+h}\right\}$ contains the edges between $v$ and the other vertices of $X_{t}$. By induction, we can assume that Table $\left(t^{\prime}\right)$ is already computed. Let us show how to compute Table $(t)$. Let $\left(L=\left(l_{1}, \cdots, l_{q+h}\right), F C=\left(f_{1}, \cdots, f_{w+1}\right), C B=\right.$ $\left.\left(b_{1}, \cdots, b_{w+1}\right)\right) \in \mathcal{F}_{t}$, there is a quasi-labelling of $G_{t}$ compatible with $(L, F C, C B)$ only if the following three conditions (easy to compute) are satisfied.

- The final colour $f_{w+1}$ that corresponds to $v$ is not in conflict with the final colours that correspond to the neighbors of $v$ in $X_{t}$. That is, for each $j \in[1, w]$ such that $v_{j} \in N_{t}(v) \cap X_{t}$, $f_{w+1} \neq f_{j}$.

- Since $v$ is introduced in $X_{t}, N_{t}(v) \backslash X_{t}=\emptyset$ and as a consequence, $c(v)$ cannot have any contribution coming from $G_{t} \backslash X_{t}$. That is $b_{w+1}=0$.

- The colour of each vertex in $G_{t}$ cannot exceed the final colour that corresponds to it. That is, for every $v_{i} \in X_{t}, b_{i}+\sum_{j=1}^{q+h} l_{j} \mathbb{I}_{i, j} \leq f_{i}$, where $\mathbb{I}_{i, j}= \begin{cases}1, & \text { if } \exists w \in N\left(v_{i}\right) \cap X_{t} \text { s.t. } w v_{i}=e_{j} \\ 0, & \text { otherwise }\end{cases}$

If one of these three conditions is not satisfied, then $\alpha_{t}(L, F C, C B)=\infty$. Otherwise, let us set

$$
\alpha_{t}(L, F C, C B)=\alpha_{t^{\prime}}\left(\left.L\right|_{X_{t^{\prime}}},\left.F C\right|_{X_{t^{\prime}}},\left.C B\right|_{X_{t^{\prime}}}\right)+\sum_{j=q+1}^{q+h} l_{j} .
$$

Following the above process, $\left((L, F C, C B), \alpha_{t}(L, F C, C B)\right)$ is added to Table $(t)$ for every $(L, F C, C B) \in \mathcal{F}_{t}$.

Let $t$ be a forget node, $t^{\prime}$ be its unique child, let $w=\left|X_{t}\right|$, and let $v$ be such that $X_{t^{\prime}}=$ $X_{t} \cup\{v\}=\left\{v_{1}, \cdots, v_{w}, v_{w+1}=v\right\}$. Moreover, let $E\left(X_{t^{\prime}}\right)=\left\{e_{1}, \ldots, e_{q}, e_{q+1}, \ldots, e_{q+h}\right\}$, where $E\left(X_{t}\right)=\left\{e_{1}, \ldots, e_{q}\right\}$. By induction, we can assume that Table $\left(t^{\prime}\right)$ is already computed. Let us show how to compute Table $(t)$.

Let $(L, F C, C B) \in \mathcal{F}_{t}$. Let $\Gamma_{t^{\prime}}$ be the subset of $\mathcal{F}_{t^{\prime}}$ that consists of all $\left(L^{\prime}, F C^{\prime}, C B^{\prime}\right) \in \mathcal{F}_{t^{\prime}}$ such that $(L, F C, C B)=\left(\left.L^{\prime}\right|_{X_{t}},\left.F C^{\prime}\right|_{X_{t}},\left.C B^{\prime}\right|_{X_{t}}\right)$ (i.e., $(L, F C, C B)$ must be the restriction to $X_{t}$ 
of some $\left.\left(L^{\prime}, F C^{\prime}, C B^{\prime}\right) \in \mathcal{F}_{t^{\prime}}\right)$ and such that $f_{w+1}^{\prime}=b_{w+1}^{\prime}+\sum_{q+1 \leq j \leq q+h} l_{j}^{\prime}$. The latter condition allows to respect the property of quasi-labelling. Since, $v \in G_{t}-X_{t}$, the "colour" that it received so far (with a contribution $b_{w+1}^{\prime}$ from the vertices in $G_{t^{\prime}}-X_{t^{\prime}}$ plus the labels $l_{q+1}^{\prime}, \cdots, l_{q+h}^{\prime}$ of its incident edges in $X_{t^{\prime}}$ must equal its "final" colour $f_{w+1}^{\prime}$. Finally, let

$$
\alpha_{t}(L, F C, C B)=\min _{\gamma \in \Gamma_{t^{\prime}}} \alpha_{t^{\prime}}(\gamma)
$$

Following the above process, $\left((L, F C, C B), \alpha_{t}(L, F C, C B)\right)$ is added to Table $(t)$ for every $(L, F C, C B) \in \mathcal{F}_{t}$.

Let $t$ be a join node, $t^{\prime}$ and $t^{\prime \prime}$ be its two children, with $X_{t}=X_{t^{\prime}}=X_{t^{\prime \prime}}=\left\{v_{1}, \ldots, v_{w}\right\}$ and $E\left(X_{t}\right)=E\left(X_{t^{\prime}}\right)=E\left(X_{t^{\prime \prime}}\right)=\left\{e_{1}, \ldots, e_{q}\right\}$. By induction, we can assume that Table $\left(t^{\prime}\right)$ and Table $\left(t^{\prime \prime}\right)$ have already been computed. Let us show how to compute Table $(t)$.

Let $(L, F C, C B) \in \mathcal{F}_{t}$. Let $\Gamma_{t}$ be the set of pairs $\left(\left(L, F C, C B^{\prime}\right),\left(L, F C, C B^{\prime \prime}\right)\right)$ such that $\left(L, F C, C B^{\prime}\right) \in \mathcal{F}_{t^{\prime}}$ and $\left(L, F C, C B^{\prime \prime}\right) \in \mathcal{F}_{t^{\prime \prime}}$ such that $C B=C B^{\prime}+C B^{\prime \prime}$ (meaning that for each $j \in[1, w], b_{j}=b_{j}^{\prime}+b_{j}^{\prime \prime}$ where $b_{j} \in C B, b_{j}^{\prime} \in C B^{\prime}$ and $\left.b_{j}^{\prime \prime} \in C B^{\prime \prime}\right)$. Then, let

$$
\alpha_{t}(L, F C, C B)=\min _{\left(\left(L, F C, C B^{\prime}\right),\left(L, F C, C B^{\prime \prime}\right)\right) \in \Gamma_{t}} \alpha_{t^{\prime}}\left(L, F C, C B^{\prime}\right)+\alpha_{t^{\prime \prime}}\left(L, F C, C B^{\prime \prime}\right)-\sum_{i=1}^{q} l_{i} .
$$

Following the above process, $\left((L, F C, C B), \alpha_{t}(L, F C, C B)\right)$ is added to Table $(t)$ for every $(L, F C, C B) \in \mathcal{F}_{t}$.

In all cases, it can be shown by induction that $\alpha_{t}(L, F C, C B) \neq \infty$ if and only if there is a quasi-labelling of $G_{t}$ compatible with $(L, F C, C B)$, and moreover, if $\alpha_{t}(L, F C, C B)<\infty$, it is the minimum sum of the edges' labels among all quasi-labellings of $G_{t}$ compatible with $(L, F C, C B)$.

\section{Particular behaviours of the problem}

In this section, we study some behaviours of the problem of determining $\operatorname{mE}_{k}(G)$ for some integer $k$ and nice graph $G$. We start by establishing that there is no systematic relationship between the proper labellings we are interested in and those considered in [1] and [5]. We then prove that, in general, using large labels might be needed for designing a labelling $\ell$ verifying $\sigma(\ell)=\operatorname{mE}(G)$. This actually remains true in cases where $G$ is a tree.

\subsection{Minimizing the maximum colour versus minimizing the sum of labels}

As described in the introductory section, quite recently the authors of 5 investigated proper labellings that minimize the maximum resulting vertex colour. The formal definitions are as follows. For a given graph $G$ and labelling $\ell$ of $G$, let $\operatorname{mS}(G, \ell)$ denote the maximum vertex colour $c_{\ell}(v)$ by $\ell$ over all vertices $v$ of $G$. For a given $k \geq \chi_{\Sigma}(G)$, let $\operatorname{mS}_{k}(G)$ denote the smallest value of $\operatorname{mS}(G, \ell)$ over all proper $k$-labellings of $G$. Now, the main parameter of interest is $\operatorname{mS}(G)$, which is defined as the minimum value of $\operatorname{mS}_{k}(G)$ over all values of $k \geq \chi_{\Sigma}(G)$.

As established in Observation 2.2 determining $\mathrm{mE}(G)$ for a nice graph $G$ can equivalently be seen as finding a proper labelling of $G$ that minimizes the sum of resulting vertex colours. Thus, one could think that maybe $\mathrm{mE}(G)$ is a good approximation of $\mathrm{mS}(G)$, or vice-versa. In this section, we show that this is actually not the case.

The next result shows that, when constructing a labelling $\ell$ of a graph $G$ with $\operatorname{mS}(G, \ell)=$ $\operatorname{mS}(G)$, we might have $\sigma(\ell)$ being arbitrarily far from $\operatorname{mE}(G)$. In other words, minimizing the maximum colour does not imply minimizing the sum of labels. This actually remains true for trees.

Theorem 4.1. There exist nice trees $T$ with arbitrarily large maximum degree $\Delta \geq 2$ for which, for any labelling $\ell$ achieving $\mathrm{mS}(T, \ell)=\mathrm{mS}(T)$, the value $\mathrm{mE}(T, \ell)$ is arbitrarily larger than $\mathrm{mE}(T)$. In particular, $\mathrm{mE}(T, \ell)=\mathrm{mE}(T)+\Delta-2$. 


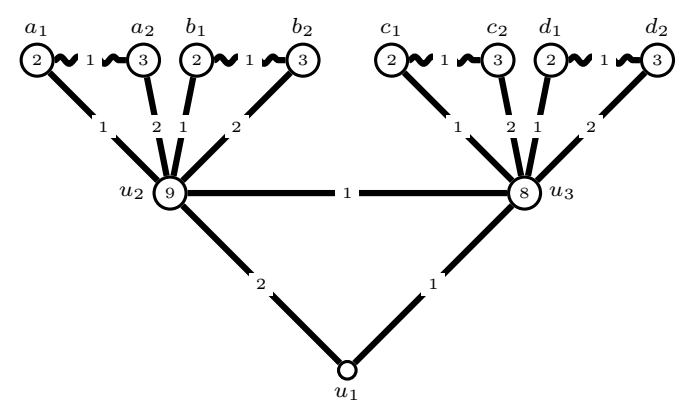

(a) $\left\{c_{\ell_{1}}\left(u_{2}\right), c_{\ell_{1}}\left(u_{3}\right)\right\}=\{8,9\}$.

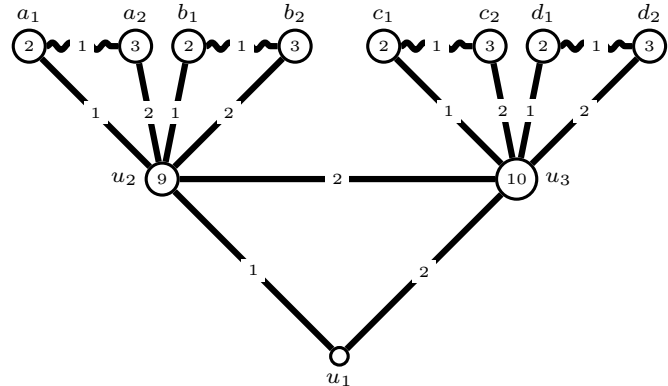

(b) $\left\{c_{\ell_{2}}\left(u_{2}\right), c_{\ell_{2}}\left(u_{3}\right)\right\}=\{9,10\}$.

Figure 1: The only proper 2-labellings $\ell_{1}$ and $\ell_{2}$ of the $T_{2}$ gadget, used in the construction illustrated in Figure 2. The induced colours for each labelling are represented as integers in the corresponding vertices. Vertex $u_{1}$ is called the root of the gadget. Wiggly edges are edges that could be labelled either 1 or 2 .

Proof. Consider the following tree $T$ with maximum degree $\Delta \geq 2$. We start from a vertex $v$ with $\Delta$ neighbours $u_{1}, \ldots, u_{\Delta}$, each of which is adjacent to $\Delta-1$ leaves. In other words, all neighbours of $v$ have degree $\Delta$, and all other vertices are leaves at distance exactly 2 from $v$.

Now consider a labelling $\ell$ of $T$ that minimizes the maximum colour, i.e., $\operatorname{mS}(T, \ell)=\operatorname{mS}(T)$. Since $T$ has adjacent vertices with degree $\Delta$, we have $\operatorname{mS}(T, \ell) \geq \Delta+1$. One possible way to attain $\mathrm{mS}(T, \ell)=\Delta+1$ is to have all edges incident to $v$ being labelled 1 , and, for each $u_{i}$, to have exactly one incident edge going to a leaf being labelled 2 and all other $\Delta-2$ incident edges being labelled 1 . Indeed, we get $c_{\ell}(v)=\Delta \neq \Delta+1=c_{\ell}\left(u_{i}\right)$ for every $i=1, \ldots, \Delta$. Actually, this is the only way to have $\operatorname{mS}(T, \ell)=\Delta+1$, because if we label the edges incident to $v$ so that $c_{\ell}(v)=\Delta+1$, then it is easy to see that the vertex $u_{i}$ such that $\ell\left(v u_{i}\right)=2$ would get $c_{\ell}\left(u_{i}\right) \geq \Delta+2$ to avoid a colour conflict between $v$ and $u_{i}$. Therefore, there is only one general way to label (actually 2-label) $T$ so that $\mathrm{mS}(T, \ell)=\mathrm{mS}(T)=\Delta+1$, and we note that the number of edges labelled 2 by $\ell$ is exactly $\Delta$ (one for each $u_{i}$ ). Thus $\mathrm{mE}(T, \ell)=|E(T)|+\Delta$.

On the other hand, we note that, regardless of the value of $\Delta$, the 2-labelling $\ell^{*}$ of $T$ where $\ell^{*}\left(v u_{1}\right)=\ell^{*}\left(v u_{2}\right)=2$ and all other edges are labelled 1 is proper. This is because we get $c_{\ell^{*}}(v)=\Delta+2, c_{\ell^{*}}\left(u_{1}\right)=c_{\ell^{*}}\left(u_{2}\right)=\Delta+1$, and $c_{\ell^{*}}\left(u_{i}\right)=\Delta$ for $i=3, \ldots, \Delta$. Thus, $\operatorname{mE}(T) \leq$ $\mathrm{mE}_{2}(T) \leq \mathrm{mE}\left(T, \ell^{*}\right)=|E(T)|+2$, and the difference between $\sigma(\ell)$ and $\sigma\left(\ell^{*}\right)$ then gets arbitrarily large as $\Delta$ grows larger.

The next result shows that the converse is also true: a labelling that minimizes the sum of labels does not necessarily minimize the maximum colour as well.

Theorem 4.2. There exist nice graphs $G$ with arbitrarily large maximum degree $\Delta \geq 12$ for which, for any 2-labelling $\ell$ achieving $\sigma(\ell)=\mathrm{mE}_{2}(G)$, the value $\mathrm{mS}(G, \ell)$ is arbitrarily larger than $\mathrm{mS}_{2}(G)$.

Proof. In [5] is exhibited a spreading gadget $G^{\curlywedge}$ (a slight modification of the graph depicted in Figure 2) with one input (the edge $u_{1} u_{2}$ ) and two outputs (the edges $u_{9} u_{10}$ and $u_{12} u_{13}$ ), that are pending edges that must all be assigned the same label (which can be either 1 or 2) by any proper 2-labelling (see Section 4.4 in [5] for details). Having a closer look at $G^{\curlywedge}$, we note that, regarding our minimization problem, it has the following property:

Claim 4.3. Let $\ell$ be a proper 2-labelling of $G^{\curlywedge}$ achieving $\sigma(\ell)=\mathrm{mE}_{2}\left(G^{\curlywedge}\right)$. Then $\ell$ assigns label 2 to the input and two outputs of $G^{\curlywedge}$.

Proof of the claim. As mentioned above, the proper 2-labellings of $G^{\curlywedge}$ are of two kinds: those $\ell_{1}$ assigning label 1 to the input and two outputs, and those $\ell_{2}$ assigning label 2 to the input and two outputs. Such labellings $\ell_{1}$ and $\ell_{2}$ are as described in Figure 2 (a) and (b), respectively (see [5]). In particular, it is important to mention that the solid edges in the figures must be labelled as illustrated (up to symmetry), while the only sources of freedom we have are the labels assigned to the wiggly edges, which can each freely be chosen to be 1 or 2 . 


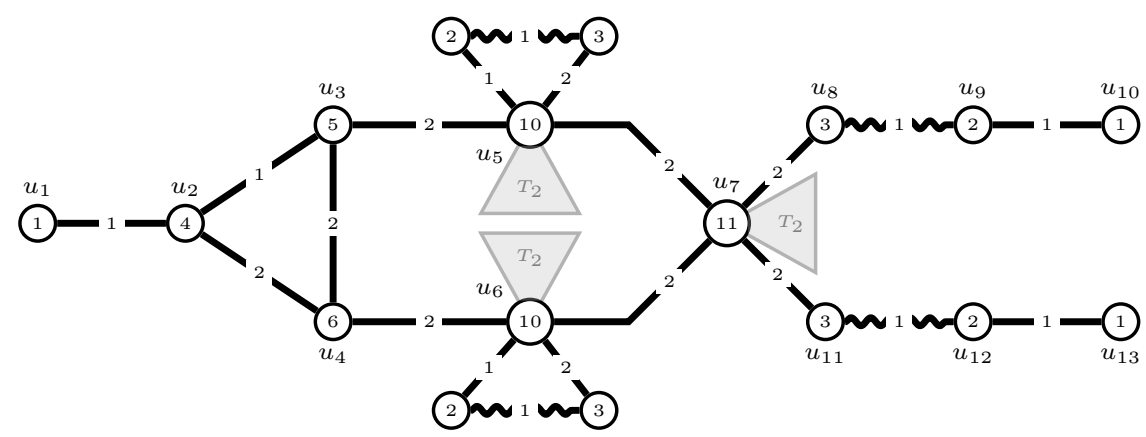

(a) Input is labelled 1.

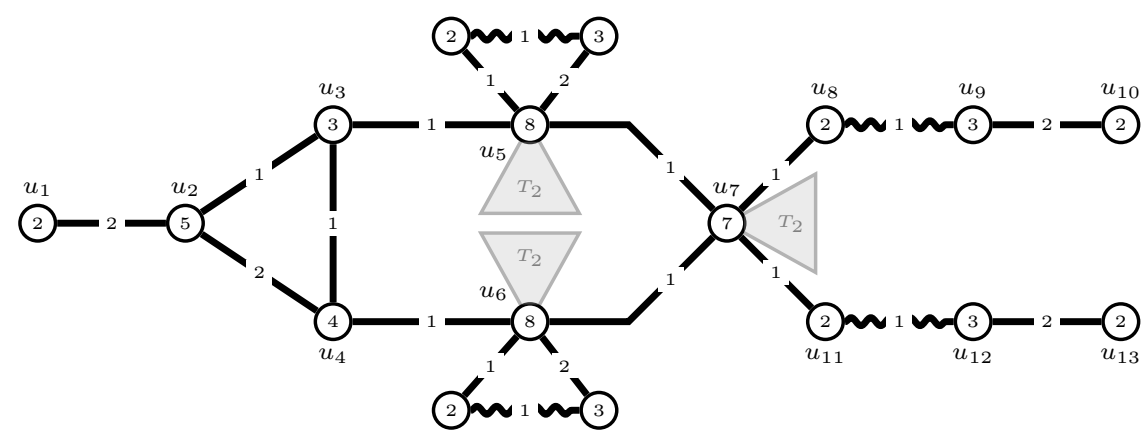

(b) Input is labelled 2 .

Figure 2: The only proper 2-labellings of the spreading gadget $G^{\curlywedge}$. A triangle marked as " $T_{2}$ " indicates that a copy of the $T_{2}$ gadget (depicted in Figure 1) is attached via its root vertex. That is, $u_{5}$ (resp., $u_{6}$ ) is identified to the roots of two copies of $T_{2}$, while $u_{7}$ is identified to the root of one copy of $T_{2}$. The induced colours for each labelling are represented as integers in the corresponding vertices. Wiggly edges are edges that could be labelled either 1 or 2 .

Let us now determine the minimum sum of labels assigned by these labellings:

- For a labelling $\ell_{1}$ of $G^{\curlywedge}$ assigning label 1 to the input and two outputs, we note, as illustrated in Figure 2 (a), that $u_{5}$ and $u_{6}$ must get colour 10, which is possible only if the two copies of $T_{2}$ attached to $u_{5}$ and $u_{6}$ are labelled as in Figure 1 (a). The vertex $u_{7}$ must get colour 11, which is not prevented by any of the two ways of labelling the copy of $T_{2}$ attached to it. Thus, for $\ell_{1}$ to minimize the sum of labels, the copies of $T_{2}$ attached to $u_{5}$ and $u_{6}$ must be labelled as in Figure 1 (a), and the copy of $T_{2}$ attached to $u_{7}$ must be labelled as in Figure 1 (a) as well (as the sum of labels in the labelling of Figure 1 (b) is larger). All wiggly edges should be assigned label 1 . In total, a minimum $\ell_{1}$ assigns label 1 to 40 edges and label 2 to 25 edges, and thus $\sigma\left(\ell_{1}\right)=90$.

- By similar arguments, we deduce that, by a labelling $\ell_{2}$ of $G^{\curlywedge}$ assigning label 2 to the input and two outputs, the copies of $T_{2}$ attached to $u_{5}$ and $u_{6}$ should be labelled as depicted in Figure 1 (b), while the copy of $T_{2}$ attached to $u_{7}$ should be labelled as depicted in Figure1 (a) (in particular, the two labellings of $T_{2}$ depicted in Figure 1 comply with $u_{7}$ having colour 7 ). Again, the wiggly edges should be labelled 1. In total, a minimum $\ell_{2}$ assigns label 1 to 42 edges and label 2 to 23 edges, and thus $\sigma\left(\ell_{2}\right)=88$.

Thus, a labelling $\ell$ of $G^{\curlywedge}$ achieving $\sigma(\ell)=\mathrm{mE}_{2}\left(G^{\curlywedge}\right)$ must assign label 2 to the input and two outputs.

Now consider the graph operation consisting in taking two copies $G_{1}$ and $G_{2}$ of $G^{\curlywedge}$, and identifying the input of $G_{1}$ and one output of $G_{2}$. As described in [5, this results in a graph $G$ with one input and three outputs such that, for every proper 2-labelling, the input and three outputs must all be assigned the same label (which can be either 1 or 2). By repeating this operation with more and more copies of $G^{\curlywedge}$, we can come up with a graph $G_{1 / 2}$ with one input and arbitrarily 


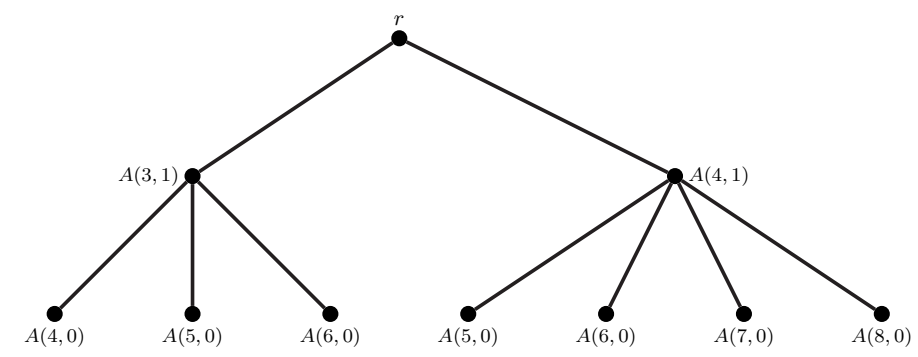

Figure 3: The auxiliary graph $A(2,2)$

many outputs that must all be assigned the same label (either 1 or 2) by a proper 2-labelling. So there are, essentially, two groups of proper 2-labellings of $G_{1 / 2}$ : those $\ell_{1}$ assigning label 1 to the input and all outputs, and those $\ell_{2}$ assigning label 2 to the input and all outputs. Clearly, a proper 2-labelling $\ell$ of $G_{1 / 2}$ verifying $\sigma(\ell)=\mathrm{mE}_{2}\left(G_{1 / 2}\right)$, when restricted to any constituting copy of $G^{\curlywedge}$ in $G_{1 / 2}$, should also be minimum in terms of sum of assigned labels. From Claim 4.3. we thus deduce that a proper 2-labelling $\ell$ of $G_{1 / 2}$ achieving $\sigma(\ell)=\mathrm{mE}_{2}\left(G_{1 / 2}\right)$ must assign Tabel 2 to the input and all outputs, i.e., must belong to the $\ell_{2}$ group mentioned above. In particular, the difference between the sum of labels of a minimum $\ell_{1}$ and the sum of labels of a minimum $\ell_{2}$ gets larger as the number of copies involved in the construction of $G_{1 / 2}$ gets larger.

Now let $G$ be the graph obtained as follows. Consider the graph $G_{1 / 2}$ with $\Delta$ outputs (for any $\Delta \geq 12$ ), and identify the degree-1 vertices of these $\Delta$ outputs to a single vertex $o^{*}$ (with maximum degree $\Delta$, as it can be checked from Figures 1 and 2 that all other vertices of $G_{1 / 2}$ have degree at most 6 ). Note that a proper 2-labelling of $G$ is also proper for $G_{1 / 2}$, since vertices of degree 1 cannot be involved in conflicts. Also, a proper 2-labelling of $G_{1 / 2}$ must proper for $G$, since $o^{*}$ has degree at least 12 while all its neighbours have degree 2. By these arguments, a proper 2-labelling $\ell$ of $G$ verifying $\sigma(\ell)=\mathrm{mE}_{2}(G)$ must thus be one of these 2-labellings $\ell_{2}$ assigning label 2 to the input and all outputs. Such an $\ell_{2}$ verifies $c_{\ell_{2}}\left(o^{*}\right)=2 \Delta$. On the other hand, a proper 2-labelling $\ell_{1}$ assigning label 1 to all outputs verifies $c_{\ell_{1}}\left(o^{*}\right)=\Delta$. Thus, a proper 2 -labelling $\ell$ of $G$ verifying $\sigma(\ell)=\mathrm{mE}_{2}(G)$ will make $o^{*}$ get colour $2 \Delta$, while there are proper 2-labellings by which $o^{*}$ gets colour $\Delta$. Note in particular that by our choice of $\Delta$, vertex $o^{*}$ must indeed be the vertex with the largest colour, as its degree is at least 12, all other vertices have degree at most 6 , and we are only assigning labels 1 and 2 .

\subsection{Using larger labels can be arbitrarily better}

In this section, we present, for any $k \geq 3$, a construction for a tree $T_{k}$ such that we have $\mathrm{mE}_{2}\left(T_{k}\right)=$ $\mathrm{mE}_{3}\left(T_{k}\right)=\mathrm{mE}_{4}\left(T_{k}\right)=\cdots=\mathrm{mE}_{k}\left(T_{k}\right)$ and $\mathrm{mE}_{k+1}\left(T_{k}\right)<\mathrm{mE}_{k}\left(T_{k}\right)$. In other words, for these trees $T_{k}$ we need to consider large labels to design a proper labelling $\ell$ achieving $\operatorname{mE}\left(T_{k}, \ell\right)=\operatorname{mE}\left(T_{k}\right)$.

Let us first introduce the auxiliary graph $A(\alpha, \beta)$ (for $\alpha \geq 2$ and $\beta \geq 0$ ), which will serve as the building block for $T_{k}$. This auxiliary graph is a tree and its built recursively as follows: For any $\alpha^{*} \in \mathbb{N}, A\left(\alpha^{*}, 0\right)$ is defined as a leaf. For any $\beta>0, A(\alpha, \beta)$ is a tree of height $\beta$, rooted in a vertex $r$ that has $\alpha$ children. For each $1 \leq i \leq \alpha$, let $c_{i}$ be the corresponding child of $r$; each $c_{i}$ is the root of a $A(\alpha+i, \beta-1)$ tree and thus $d\left(c_{i}\right)=\alpha+i+1$ (since each $c_{i}$ has $\alpha+i$ children of its own as well as an edge connecting him with his parent). Note that $d\left(c_{i}\right) \in D(\alpha):=[\alpha+2,2 \alpha+1]$ and that for $i \neq j, d\left(c_{i}\right) \neq d\left(c_{j}\right)$ (and thus all the values of $D(a)$ are used exactly once). Finally, we say that $A(\alpha, \beta)$ is represented by $r$. The auxiliary graph $A(2,2)$ is illustrated in Figure 3 .

Let us also define the pending auxiliary graph that corresponds to $A(\alpha, \beta)$ as $P(\alpha, \beta)=(V, E)$, where $V=V(A(\alpha, \beta)) \cup\{v\}$ and $E=E(A(\alpha, \beta)) \cup v r$; in essence $P(\alpha, \beta)$ is $A(\alpha, \beta)$ with an extra vertex $v$ connected to $r$. We say that $P(\alpha, \beta)$ is pending from $v$. Observe that $P(\alpha, \beta)$ is locally irregular and thus the labelling $\ell$ that assigns label 1 on every one of its edges is proper and $\mathrm{mE}(P(\alpha, \beta), \ell)=|E|$.

Lemma 4.4. Let $\beta \in \mathbb{N}^{*}$ and $\alpha \geq 2$. Let $\ell$ be an $\alpha$-labelling of the pending auxiliary graph $P(\alpha, 2 \beta)$ pending from $v$. Let $u, w \in V(P(\alpha, 2 \beta))$ such that $1 \leq \operatorname{dist}(u, v) \leq 2$ and $w$ is the parent of $u$. If $\ell(u w)>1$, then $\operatorname{mE}(P(\alpha, 2 \beta), \ell) \geq|E|+\beta$. 
Proof. Let us prove the Lemma for the case where $\operatorname{dist}(u, v)=2$ and $u$ is the root of the $A(\alpha+$ $1,2 \beta-1$ ) subtree, i.e., $u=r$ and $w=v$ (similar arguments hold for the other cases) and let us first assume that $u w$ is the only edge of $P(\alpha, \beta)$ that has label more than 1 , say $\ell(u w)=\alpha^{\prime}$ where $2 \leq \alpha^{\prime} \leq \alpha$. It follows that $c_{\ell}(u)=\alpha+\alpha^{\prime}+1$ and that $\alpha+3 \leq c_{\ell}(u) \leq 2 \alpha+1$. Since all edges of $P(\alpha, 2 \beta)$ except $u w$ is labelled 1, each child $y$ of $u$ has $c_{\ell}(y)=d(y)$. Moreover, since $u$ is the root of the $A(\alpha+1,2 \beta-1)$ tree, each one of the $\alpha+1$ children of $u$ has a unique degree in the set $D(\alpha+1)$. But $D(\alpha+1)=[\alpha+3,2 \alpha+2]$ and $c_{\ell}(u) \in D(\alpha+1)$. It follows that there exists a child of $u$ that has the same induced, by $\ell$, colour as $u$. Thus $\ell$ must assign a label different than 1 to at least one more edge of $P(\alpha, \beta)$, and the argument can be repeated at least $\beta$ times (since the height of $T(\alpha, 2 \beta)$ is $2 \beta+1)$, leading to $\ell$ having to assign a label different than 1 to at least $\beta$ edges. The exact value of $\operatorname{mE}(P(\alpha, 2 \beta), \ell)=|E|+\beta$ is reached if each time the argument is repeated, $\alpha^{\prime}=2$ and the next edge that gets assigned label 2 is at distance 2 from the previous.

Theorem 4.5. For every $k \geq 2$, there exists a nice graph $T_{k}$ such that $\mathrm{mE}_{k+1}\left(T_{k}\right)<\mathrm{mE}_{k}\left(T_{k}\right)$.

Proof. Let $k \geq 2$ and let us describe the construction of $T_{k}=(V, E)$. For $0 \leq j \leq k-1$, let $P(k+j, 2(k+1))$ be the auxiliary graph pending from $v_{j}$ that corresponds to an auxiliary graph $A(k+j, 2(k+1))$ (represented by a vertex $r_{j}$ ) and let $u, v$ be two vertices connected by the edge $u v$. The tree $T_{k}$ is the graph that is produced by identifying $v$ with each one of the $v_{j}$. Observe that since $r_{j}$ represents $A(k+j, 2(k+1))$, each $r_{j}$ has $d\left(r_{j}\right)=k+j+1$ in $T_{k}$ and that the height of $T_{k}$ is $2(k+1)+1$. Also observe that in $T_{k}$, since $N(v)=\left\{r_{0}, \ldots, r_{k-1}, u\right\}, d(v)=k+1=d\left(r_{0}\right)$.

Claim 4.6. There exists a $(k+1)$-labelling $\ell$ of $T_{k}$ such that $\sigma(\ell)=|E|+k$.

Proof of the claim. Note that $T_{k}$ is almost locally irregular. Indeed, let $w$ be a non leaf vertex of $T_{k}$, other than $r_{1}, v$ and $u$, and let $x$ be its parent. If $d(w)=d+1$ then $d+1>d(x)$ (by construction) and $w$ has $d$ children $c h_{1}, \ldots, c h_{d}$, each one having degree at least $d+2$. In fact, the only adjacent vertices that have the same degree are $v$ and $r_{0}$.

Let $\ell$ be the $k+1$-labelling of $T_{k}$ that assigns label $k+1$ on the edge $u v$ and label 1 on the remaining edges of $T_{k}$. Then $c_{\ell}(v)=2 k+1$ and for each $0 \leq i \leq k-1, d\left(r_{j}\right) \in[k+1,2 k]$ and thus there is no conflict between the colour of $v$ and that of its children. It follows that $\ell$ is a proper $(k+1)$-labelling for $T_{k}$ and $\sigma(\ell)=|E|+k$.

Let $\ell^{\prime}$ be any proper $k$-labelling of $T_{k}$. It suffices to show that $\sigma\left(\ell^{\prime}\right)>|E|+k$. Let $w \in N\left(r_{0}\right) \backslash\{v\}$ and $y \in N(v) \backslash\left\{u, r_{0}\right\}$. Once more, since $d(v)=d\left(r_{0}\right)=k+1$, at least one of the edges $u v, r_{0} w$ or $v y$ has to have a label different than 1 for $\ell^{\prime}$ to be proper. Let $\ell^{\prime}(u v)=l$ with $2 \leq l \leq k$ and assume that only this is the only edge of $T_{k}$ that has a label different than 1 . Then $c_{\ell^{\prime}}(v)=k+l$ and $k+l \in[k+2,2 k]$. Recall that for each $0 \leq j \leq k-1, r_{j}$ has $d\left(r_{j}\right)=k+j+1$ and thus $d\left(r_{j}\right) \in[k+1,2 k]$ and since $u v$ is the only edge with a label different than $1, c_{\ell^{\prime}}\left(r_{j}\right)=d\left(r_{j}\right)$. It follows that there exists a $j \in[0, k-1]$ such that $c_{\ell^{\prime}}\left(r_{j}\right)=c_{\ell^{\prime}}(v)$ leading to $\ell^{\prime}$ not being proper. Thus there must exist another edge $u^{\prime} v^{\prime}$ (with, say, $u^{\prime}$ being the parent of $v^{\prime}$ ) that is assigned a label different than 1 by $\ell^{\prime}$. This edge, however, belongs to $P(q, 2(k+1))$ (for some $q \in[k, 2 k-1]$ ) and have $1 \leq \operatorname{dist}\left(v^{\prime}, v\right) \leq 2$. It follows from Lemma 4.4 that $\mathrm{mE}\left(T_{k}\right) \geq|E|+k+1$. The cases where $r_{0} w$ or $v y$ are assigned a label different than 1 follow by applying directly Lemma 4.4 .

Observe that the height of $T_{k}$ can be controlled by changing the $\beta$ value of the pending auxiliary graphs that form it. Furthermore, it follows from Lemma 4.4 that $\mathrm{mE}\left(T(\alpha, 2 \beta)<\operatorname{mE}\left(T\left(\alpha, 2 \beta^{\prime}\right)\right)\right.$ for $\beta<\beta^{\prime}$. This proves the following corollary:

Corollary 4.7. For every $k \geq 2$, there exists a graph $T_{k}$ such that $\mathrm{mE}_{k+1}\left(T_{k}\right)$ is arbitrarily smaller than $\mathrm{mE}_{k}\left(T_{k}\right)$.

\section{Bounds}

Observation 2.1 establishes that, for any nice graph $G$, in general $\mathrm{mE}(G)$ should be expressed as a function of $|E(G)|$. To date, the best result towards the 1-2-3 Conjecture, due to Kalkowski, Karoński and Pfender [11, states that $\chi_{\Sigma}(G) \leq 5$ holds for every nice graph $G$. It implies the following: 
Theorem 5.1 ([11]). For every nice graph $G$, we have $\mathrm{mE}(G) \leq \mathrm{mE}_{5}(G) \leq 5|E(G)|$.

Of course, the upper bound in Theorem 5.1 is immediately improved for every nice graph $G$ for which we can beat the upper bound of Kalkowski, Karoński and Pfender. In particular, let us recall that $\chi_{\Sigma}(G) \leq 3$ whenever $\chi(G) \leq 3$ (see [12]), which implies that $\mathrm{mE}(G) \leq \mathrm{mE}_{3}(G) \leq 3|E(G)|$ holds here. Recently, Przybyło proved in [15] that $\chi_{\Sigma}(G) \leq 4$ whenever $G$ is regular, which implies that $\mathrm{mE}(G) \leq \mathrm{mE}_{4}(G) \leq 4|E(G)|$ holds. More results of this sort can be found e.g. in the survey [16] by Seamone.

More generally, the 1-2-3 Conjecture, if true, would imply that, for every nice graph $G$, we even have $\mathrm{mE}(G) \leq \mathrm{mE}_{3}(G) \leq 3|E(G)|$. A natural question to wonder is whether there exist graphs $G$ for which $\mathrm{mE}(G)$ is close to this theoretical upper bound $3|E(G)|$. Recall that, already, we have $\mathrm{mE}(G) \leq \mathrm{mE}_{2}(G) \leq 2|E(G)|$ whenever $\chi_{\Sigma}(G) \leq 2$. The fact that we are not aware of graphs $G$ with $\chi_{\Sigma}(G)=3$ needing a lot of 3's in any proper 3-labelling leads us to suspect that even the following conjecture might be true.

Conjecture 5.2. For every nice graph $G$, we have $\mathrm{mE}(G) \leq 2|E(G)|$.

Conjecture 5.2 holds true for all graphs $G$ with $\chi_{\Sigma}(G) \leq 2$, recall Observation 2.1 Experimentation via computer programs led us to observe that, actually, it might even be true that the equality $\mathrm{mE}(G)=2|E(G)|$ holds if and only if $G$ is $K_{3}$ or $C_{6}$, recall Theorems 2.5 and 2.6 However, these cases are very peculiar, due to the small number of edges these two graphs have.

Throughout this section, we provide weaker results towards Conjecture 5.2 for graphs with given chromatic number, we verify Conjecture 5.2 for bipartite graphs, and we prove a stronger result in the particular case of trees.

\subsection{Graphs with large chromatic number}

Towards Conjecture 5.2 , we provide a general bound on $\operatorname{mE}(G)$ being a function of the chromatic number $\chi(G)$. In particular, the bound we get is better than that in Theorem 5.1 and even better than the conjectured one in Conjecture 5.2 , for dense enough graphs.

In the upcoming proofs, we make use of arguments that are fairly common in this field (see e.g. [1, 4, 7, 12, 13, 17, 18]), based on label modifications along walks with certain parity length. Recall that a walk in a graph is a path in which vertices and/or edges might be repeated. A walk is said closed if it starts and ends at a same vertex. We say a path is even (odd, respectively) if it is of even length (odd length, respectively).

The next results are for graphs that are not bipartite. Results dedicated to bipartite graphs will be provided in the next section.

Theorem 5.3. Let $G=(V, E)$ be a nice graph with chromatic number $k=\chi(G)$ at least 3 . Then, we have $\operatorname{mE}(G) \leq \mathrm{mE}_{k+1}(G) \leq|E|+k|V|$.

Proof. Since $k \geq 3$, we have that $G$ is not a bipartite graph. It follows that there exists an odd cycle $C$ in $G$. Let $H$ be the subgraph of $G$ constructed according the following procedure: start by $H=C$. Then, iteratively, for each vertex $v \in V \backslash V(H)$ such that there exists a vertex $h \in V(H)$ with $v h \in E$, add $v$ to $V(H)$ and $v h$ to $E(H)$. Repeat this process until $V(H)=V$. In the end, $H$ will contain only one cycle, the cycle $C$, and simple paths that lead to all the vertices of $G$ that do not belong to $C$. Observe that for each $e \in E(C)$, the subgraph $H-e$ is a spanning tree of $G$. Thus $|E(H)|=|V(G)|$.

Let $G^{\prime}=G \cup S_{0}$, where $S_{0}=\emptyset$, and $S_{i} \subseteq V\left(G^{\prime}\right)$, for $1 \leq i \leq k$, be the $k$ stable sets induced by a proper colouring $c$ of $G^{\prime}$ (i.e. if $v \in S_{i}$ then $c(v)=i$ ). We are going to construct a $(k+1)$-labelling $\ell$ on the edges of $G^{\prime}$ such that $\sigma(\ell) \leq\left|E\left(G^{\prime}\right)\right|+k\left|V\left(G^{\prime}\right)\right|$. Let us start by having $\ell$ assign label 1 to all edges of $G^{\prime}$. At this point, the colour of every vertex is exactly its degree. For each $0 \leq i \leq k$, let $S_{i}^{*}=\left\{v \in S_{i} \mid c_{\ell}(v)=i \bmod (k+1)\right\}$ (obviously $S_{0}^{*}=\emptyset$ ). Our goal is to modify $\ell$ so that for each $i$, we have $S_{i}^{*}=S_{i}$, from which it follows that $c_{\ell}$ is a proper colouring of $G^{\prime}$. Aiming at reaching that conclusion, note that, modulo $k+1$, we can equivalently have $\ell$ assigning labels $0, \ldots, k$ instead.

Let $v^{*} \in V\left(G^{\prime}\right)$ such that $d(v)=d(\bmod k+1)$. Since $c_{\ell}\left(v^{*}\right)=d\left(v^{*}\right)$, it follows that $v^{*} \in S_{d}^{*}$. Free to relabel the stable sets induced by $c$, we may assume that $d$ is such that $S_{d-1}=\emptyset$. For 
each $v \in S_{i} \backslash S_{i}^{*}$, we define $P_{o}(v)=\left(v^{*}, h_{1}^{o}, \ldots, h_{n}^{o}, v\right)$ and $P_{e}(v)=\left(v^{*}, h_{1}^{e}, \ldots, h_{m}^{e}, v\right)$ to be an odd and an even walk respectively, following the edges of $H$, that connect $v^{*}$ and $v$ (thus $n$ is an even number and $m$ is an odd number). These walks are sure to exist because $H$ contains the odd cycle $C$. Let $\ell^{\prime}$ be the modified labelling $\ell$. To modify $P_{o}(v)$, we alternate between removing $1 \bmod (k+1)$ and adding $1 \bmod (k+1)$ from the labels of its edges. Thus $\ell^{\prime}\left(v^{*} h_{1}^{o}\right)=$ $\ell\left(v^{*} h_{1}^{o}\right)-1 \bmod (k+1), \ell^{\prime}\left(h_{1}^{o} h_{2}^{o}\right)=\ell\left(h_{1}^{o} h_{2}^{o}\right)+1 \bmod (k+1), \ldots, \ell^{\prime}\left(h_{n}^{o} v\right)=\ell\left(h_{n}^{o} v\right)-1 \bmod (k+1)$. Similarly, to modify $P_{e}(v)$, we alternate between adding $1 \bmod (k+1)$ and removing $1 \bmod (k+1)$ from the labels of its edges. Thus $\ell^{\prime}\left(v^{*} h_{1}^{e}\right)=\ell\left(v^{*} h_{1}^{e}\right)+1 \bmod (k+1), \ell^{\prime}\left(h_{1}^{e} h_{2}^{e}\right)=\ell\left(h_{1}^{e} h_{2}^{e}\right)-$ $1 \bmod (k+1), \ldots, \ell^{\prime}\left(h_{n}^{e} v\right)=\ell\left(h_{n}^{e} v\right)-1 \bmod (k+1)$. These modifications do not affect the colours of the internal vertices of $P_{o}(v)$ and $P_{e}(v)$. We perform these modifications the one after the other. That is if we start by modifying $P_{o}(v)$, we continue by modifying $P_{e}(v)$, then with $P_{o}(v)$ and so on. Each time we modify $P_{o}(v)$ or $P_{e}(v)$, the colour of $v$ is reduced by $1 \bmod (k+1)$ and if we alternate between modifying $P_{o}(v)$ and $P_{e}(v)$, then the colour of $v^{*}$ stays the same.

Let $v \in S_{i} \backslash S_{i}^{*}$. We alternate between modifying $P_{o}(v)$ and $P_{e}(v)$ until $c_{\ell^{\prime}}(v)=i \bmod (k+1)$. Then me move on to modify another vertex $v^{\prime} \in S_{i^{\prime}} \backslash S_{i^{\prime}}^{*}\left(i\right.$ is not necessarily different than $\left.i^{\prime}\right)$. If the last modification for $v$ was on $P_{o}(v)$, we start with modifying $P_{e}\left(v^{\prime}\right)$ and once more we proceed by alternating between modification on $P_{e}\left(v^{\prime}\right)$ and $P_{o}\left(v^{\prime}\right)$ and vice versa.

It is clear that once the above process is finished, for each $v \neq v^{*}$ and $i, v \in S_{i}$ if and only if $c_{\ell^{\prime}}(v)=i \bmod (k+1)$. Also, if the total number $w$ of modifications done is even, then $c_{\ell^{\prime}}\left(v^{*}\right)=d \bmod (k+1)$ and if $w$ is odd, then $c_{\ell^{\prime}}\left(v^{*}\right)=d-1 \bmod (k+1)$. In any case, and since before the modifications $S_{d-1}=\emptyset, c_{\ell^{\prime}}$ is a proper colouring of $G$. Note that this remains true when turning all labels 0 into $k+1$, so that $\ell^{\prime}$ is a $(k+1)$-labelling as desired. Recall also that the modifications are done on the edges of $H$ and $|E(H)|=|V(G)|$. In the worst case, all the edges of $H$ are labelled $k+1$ by $\ell^{\prime}$ and thus $\operatorname{mE}\left(G, \ell^{\prime}\right) \leq|E|+k|V|$.

In most proof contexts making use of the walk-switching procedure described in the proof of Theorem 5.3 , there are favourable situations in which the bound can be further reduced. The next result illustrates that fact.

Theorem 5.4. Let $G=(V, E)$ be a nice graph with odd chromatic number $k=\chi(G)$ at least 3 . Then, we have $\mathrm{mE}(G) \leq \mathrm{mE}_{k}(G) \leq|E|+(k-1)|V|$.

Proof. Let $H$ be the subgraph of $G$ as it was defined in the proof of Theorem 5.3 and $S_{i}$ be the stable sets induced by a proper colouring $c$ of $G$. Our goal is to reach, by $\ell$, the desired colours modulo $k$. Under that assumption, we can here assign labels $0, \ldots, k-1$ instead. Once more, we start with $\ell$ assigning label 1 to all edges of $G$. For each $1 \leq i \leq k$, let $S_{i}^{*}=\left\{v \in S_{i} \mid c_{\ell}(v)=i \bmod k\right\}$.

For each $v \in S_{i} \backslash S_{i}^{*}$, let $P_{v}$ be an odd length closed walk of $H$ that contains $v$. Again the existence of $P_{v}$ is guaranteed because of $C$. We proceed by modifying the labels of $P_{v}$ : we alternate between adding 1 (modulo $k$ ) and removing 1 (modulo $k$ ) from the labels of consecutive edges of $P_{v}$. Since $P_{v}$ is a closed walk of odd length, exactly two consecutive edges (not necessarily distinct) will have to be altered in the same way (i.e. either they are both incremented by 1 or reduced by 1 modulo $k$ ). The modification is done so that these two edges have $v$ as a common vertex. Let $\ell^{\prime}$ be the modified $\ell$ and let us assume that the labels of the edges of $H$ that are adjacent to $v$ are both incremented by 1 modulo $k$ (symmetric argument holds for the other case). Clearly $c_{\ell^{\prime}}(v)=\left(c_{\ell}(v)+2\right) \bmod k$ and since $k$ is odd, by repeating this process the desired value $c_{\ell^{\prime}}(v)=i \bmod k$ is eventually reached.

Eventually turn all 0's into $k$ 's. In the worst case, $\ell^{\prime}$ assigns label $k$ on each one of the $|V|$ edges of $H$. Thus $\mathrm{mE}\left(G, \ell^{\prime}\right) \leq|E|+(k-1)|V|$.

\section{$5.2 \quad$ Bipartite graphs}

In this section, we prove Conjecture 5.2 for nice bipartite graphs. It turns out, however, that we are not aware of many bipartite graphs $G$ for which $\mathrm{mE}_{3}(G)$ reaches exactly $2|E(G)|$. To go further, we both improve the upper bound in particular contexts, and exhibit constructions of bipartite graphs $G$ with large value of $\mathrm{mE}_{2}(G)$, that are legitimate candidates for having $\mathrm{mE}(G)$ large. Throughout this section, it is worth keeping in mind that determining $\mathrm{mE}_{2}(G)$ for a given bipartite graph $G$ is $\mathcal{N} \mathcal{P}$-complete by Theorem 3.2 


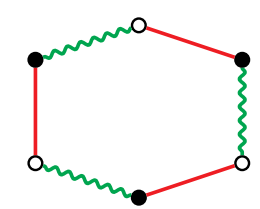

(a)

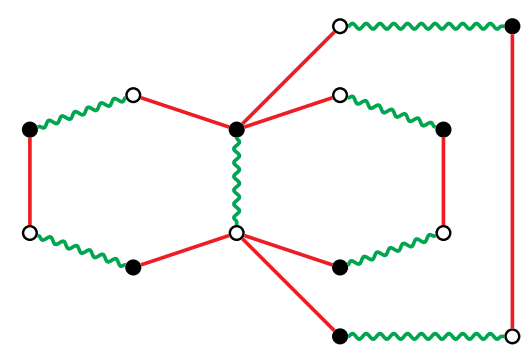

(c)

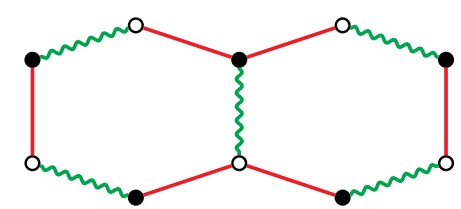

(b)

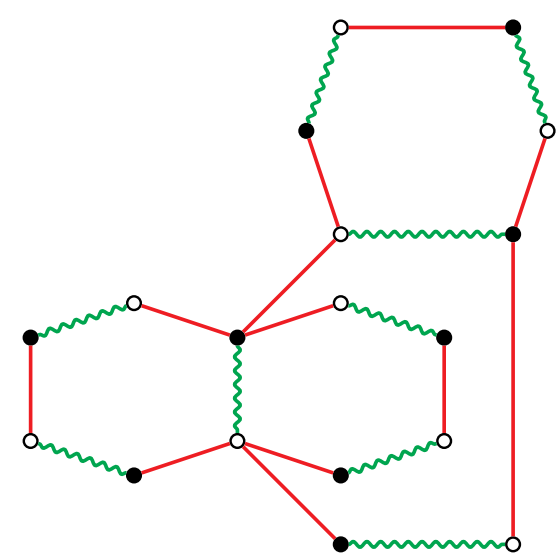

(d)

Figure 4: Constructing an odd multi-cactus through several steps, from the red-green $C_{6}$ (a). Redgreen paths with length at least 5 congruent to 1 modulo 4 are being repeatedly attached onto green edges through steps (b) to (d). Solid edges are red edges. Wiggly edges are green edges.

\subsubsection{Conjecture 5.2 for nice bipartite graphs}

Since, according to [12], nice bipartite graphs $G$ verify the 1-2-3 Conjecture and some of them even verify $\chi_{\Sigma}(G)=3$, they can be classified into three classes $\mathcal{B}_{1}, \mathcal{B}_{2}$ and $\mathcal{B}_{3}$ where, for each $i \in\{1,2,3\}$, the set $\mathcal{B}_{i}$ contains exactly the connected bipartite graphs $G$ with $\chi_{\Sigma}(G)=i$. Note that $\mathcal{B}_{1}$ contains the locally irregular bipartite graphs $G$, each one of which verifies $\mathrm{mE}_{1}(G)=|E(G)|$. The graphs $G$ of $\mathcal{B}_{2}$ admit proper 2-labellings, and, for these, by Observation 2.1 we have $\mathrm{mE}_{2}(G) \leq 2|E(G)|$. So, in order to prove Conjecture 5.2 for nice bipartite graphs, we only need to focus on the graphs of $\mathcal{B}_{3}$.

The graphs of $\mathcal{B}_{3}$ were characterized by Thomassen, Wu and Zhang in [18, who proved that these graphs are exactly the so-called odd multi-cacti, which are the graphs that can be obtained at any step of the following procedure (see Figure 4 for an illustration):

- Start from a cycle with length at least 6 congruent to 2 modulo 4 whose edges are properly coloured with red and green (i.e., no two adjacent edges have the same colour).

- Consider a green edge $u v$ and join $u$ and $v$ via a new path of length at least 5 congruent to 1 modulo 4 whose edges are properly coloured with red and green, where both the edge incident to $u$ and the edge incident to $v$ are red.

It is worth mentioning that odd multi-cacti are 2-degenerate and 2-connected. Also, they are bipartite, and both of their parts have odd size. It can also be noted that for every green edge $u v$, we have $d(u)=d(v)$, and no two green edges share ends. In [7], it was actually shown that any nice bipartite graph with a part of even size does not belong to $\mathcal{B}_{3}$ :

Lemma 5.5. [7] Let $G$ be a connected bipartite graph with bipartition $(U, V)$. If $|U|$ is even, then $\chi_{\Sigma}(G) \leq 2$. Furthermore, $G$ admits proper 2-labellings where all vertices of $U$ have odd colour while all vertices of $V$ have even colour.

We are now ready to prove our main result in this section.

Theorem 5.6. For every nice bipartite graph $G$, we have $\mathrm{mE}(G) \leq \mathrm{mE}_{3}(G) \leq 2|E(G)|$. 
Proof. Since the statement holds for $G \in \mathcal{B}_{1} \cup \mathcal{B}_{2}$, as explained earlier, we can assume $G \in \mathcal{B}_{3}$, i.e., $G$ is an odd multi-cactus with bipartition $(U, V)$ (where both $|U|$ and $|V|$ are odd). If $G$ is a cycle with length at least 6 congruent to 2 modulo 4 , then the result follows from Theorem 2.6. Thus, we may assume that $\Delta(G) \geq 3$, i.e., some path attachments were made to build $G$ starting from an original cycle.

Let us consider the last green edge $x y$ to which a path $P=\left(x, v_{1}, \ldots, v_{4 k}, y\right)$ was attached in the construction of $G$, where $k \geq 1$. Recall that $d(x)=d(y) \geq 3$ by construction.

Consider $G^{\prime}=G-\left\{v_{1}, v_{2}, v_{3}\right\}$. Assuming $v_{1}, v_{3} \in U$ and $v_{2} \in V$, the bipartition of $G^{\prime}$ is $\left(U^{\prime}, V^{\prime}\right)=\left(U \backslash\left\{v_{1}, v_{3}\right\}, V \backslash\left\{v_{2}\right\}\right)$. This means that $\left|V^{\prime}\right|$ is even. By Lemma 5.5, there is a proper 2-labelling $\ell^{\prime}$ of $G^{\prime}$ such that all vertices of $U^{\prime}$ have even colour while all vertices of $V^{\prime}$ have odd colour. Since $x \in V^{\prime}$, the colour $c_{\ell^{\prime}}(x)$ is odd, and thus at least 3 since $d_{G^{\prime}}(x) \geq 2$. Similarly, $v_{4} \in V^{\prime}$, so the colour $c_{\ell^{\prime}}\left(v_{4}\right)$ is odd, and it is precisely 1 since $d_{G^{\prime}}\left(v_{4}\right)=1$.

We now extend $\ell^{\prime}$ to a proper 3-labelling $\ell$ of $G$, by assigning label 1 to $v_{1} v_{2}$, label 2 to $x v_{1}$ and $v_{3} v_{4}$, and label 3 to $v_{2} v_{3}$. This way:

- $c_{\ell}(x)$ and $c_{\ell}\left(v_{4}\right)$ remain odd;

- $c_{\ell}\left(v_{1}\right)=3<5 \leq c_{\ell}(x)$;

- $c_{\ell}\left(v_{3}\right)=5>3=c_{\ell}\left(v_{4}\right)$;

- $c_{\ell}\left(v_{2}\right)=4 \notin\left\{c_{\ell}\left(v_{1}\right), c_{\ell}\left(v_{3}\right)\right\}=\{3,5\}$.

For these reasons, it should be clear that $\ell$ is indeed a proper 3-labelling of $G$. We additionally note that label 3 is actually assigned only once by $\ell$, to $v_{2} v_{3}$. Furthermore, $\ell$ assigns label 1 at least once, e.g. to $v_{1} v_{2}$. From this, it follows that $\sigma(\ell) \leq 2|E(G)|$.

As mentioned earlier, the only bipartite graph $G$ verifying $\operatorname{mE}(G)=2|E(G)|$ we are aware of, is $C_{6}$. Due to the small number of edges of $C_{6}$, this case looks quite pathological. In particular, it is natural to wonder whether Theorem 5.6 can be improved in general, when excluding $C_{6}$. We investigate this concern in what follows.

\subsubsection{Lower bounds for some bipartite graphs}

Our main result in this section is that, in general, for a nice bipartite graph $G$ it is not possible to lower $\mathrm{mE}_{2}(G)$ below the $\frac{3}{2}|E(G)|$ barrier. Put differently, there exist bipartite graphs for which label 2 must be assigned to at least half of the edges by any proper 2-labelling. This is a consequence of the following more general result, which is of independent interest.

Theorem 5.7. Let $G$ be any nice graph, and let $H$ be a graph obtained from $G$ by subdividing every edge e exactly $n_{e}$ times, where $n_{e}=4 k_{e}+3$ for some $k_{e} \geq 0$. Then $\chi_{\Sigma}(H)=2$. Furthermore, $\mathrm{mE}_{2}(H)=\frac{3}{2}|E(H)|$.

Proof. For every edge $e=u v$ of $G$, let us denote by $P_{e}$ the corresponding path of length $4\left(k_{e}+1\right)$ in $H$. Note that $H$ has many adjacent 2-vertices, so $\chi_{\Sigma}(H)>1$. Also, $H$ is bipartite with bipartition $X \cup Y$, where w.l.o.g. $X$ contains all vertices of $G$. Now let $\ell$ be the 2-labelling of $H$ obtained by considering every edge $e=u v$ of $G$, and assigning labels $2,1,1,2,2,1,1, \ldots, 1,1,2$ to the consecutive edges of $P_{e}$ as going from $u$ to $v$. Then $\ell$ is proper since all vertices in $X$ have even colour, while all vertices in $Y$ have odd colour. The last part of the claim follows from the fact that for every edge $e$ of $G$, in any labelling $\ell$ of $H$ every two edges of $P_{e}$ being at distance 2 apart must receive distinct labels (recall Observation 2.3). Due to the length of $P_{e}$, this implies that the sum of the labels assigned to its edges is at least $\frac{3}{2}\left|E\left(P_{e}\right)\right|$. Thus, $\sigma(\ell) \geq \frac{3}{2}|E(H)|$.

Corollary 5.8. There exist infinitely many bipartite graphs $G \in \mathcal{B}_{2}$ with various structure verifying $\mathrm{mE}_{2}(G)=\frac{3}{2}|E(G)|$. This remains true for trees.

Proof. This follows from Theorem 5.7. The last part of the statement is because any subdivision of a tree is clearly a tree itself. 
In particular through experimentation via computer programs, we also managed to come up with the following construction yielding bipartite graphs $G$ for which $\mathrm{mE}_{2}(G)$ slightly exceeds $\frac{3}{2}|E(G)|$. These graphs can be constructed as follows. Let $x, y \geq 4$ be any two integers congruent to 0 modulo 4 . The graph $H(x, y)$ is the graph obtained by starting from the disjoint union of a cycle $C$ with length $x$ and a cycle $C^{\prime}$ with length $y$, by adding an edge joining any vertex of $C$ and any vertex of $C^{\prime}$. Note that $H(x, y)$ has odd size.

Theorem 5.9. Let $x, y \geq 4$ be any two integers at least 4 congruent to 0 modulo 4 . Then, we have $\mathrm{mE}_{2}(H(x, y))=\left\lceil\frac{3}{2}|E(H(x, y))|\right\rceil$.

Proof. We begin by showing the following Claim:

Claim 5.10. Let $G$ be obtained from a cycle $C$ with length $x$ at least 4 congruent to 0 modulo 4 by adding an edge from any vertex $v$ of $C$ to a new pending vertex $u$. Then, by any proper 2-labelling $\ell$ of $G$, exactly half of the edges of $G$ must be labelled 2. Furthermore, either:

- $\ell(v u)=1$ and $c_{\ell}(v)=5$, or

- $\ell(v u)=2$ and $c_{\ell}(v)$ can be either of $4,5,6$.

Proof of the claim. Let us denote by $v_{0}, \ldots, v_{x-1}$ the successive vertices of $C$, where $v_{0}=v$. Because $d\left(v_{i}\right)=2$ for every $i \in\{1, \ldots, x-1\}$, recall, according to Observation 2.3 that, by any proper 2-labelling $\ell$ of $G$, we must have $\ell\left(v_{0} v_{1}\right) \neq \ell\left(v_{2} v_{3}\right) \neq \ell\left(v_{4} v_{5}\right) \neq \cdots \neq \ell\left(v_{x-2} v_{x-1}\right)$ (and thus, by the length of $x$, we have $\left.\ell\left(v_{0} v_{1}\right) \neq \ell\left(v_{x-2} v_{x-1}\right)\right)$, and similarly $\ell\left(v_{1} v_{2}\right) \neq \ell\left(v_{3} v_{4}\right) \neq \ell\left(v_{5} v_{6}\right) \neq$ $\cdots \neq \ell\left(v_{x-1} v_{0}\right)$ (and thus $\ell\left(v_{1} v_{2}\right) \neq \ell\left(v_{x-1} v_{0}\right)$ ). So there are essentially three ways for $\ell$ to be designed:

- If $\ell\left(v_{0} v_{1}\right)=\ell\left(v_{0} v_{k-1}\right)=1$, then $\ell\left(v_{1} v_{2}\right)=\ell\left(v_{k-1} v_{k-2}\right)=2$, and $c_{\ell}\left(v_{1}\right)=c_{\ell}\left(v_{k-1}\right)=3$. In that case, so that $c_{\ell}\left(v_{0}\right) \neq 3$, we must have $\ell\left(v_{0} u\right)=2$ in which case $c_{\ell}\left(v_{0}\right)=4$.

- If $\ell\left(v_{0} v_{1}\right)=\ell\left(v_{0} v_{k-1}\right)=2$, then $\ell\left(v_{1} v_{2}\right)=\ell\left(v_{k-1} v_{k-2}\right)=1$, and $c_{\ell}\left(v_{1}\right)=c_{\ell}\left(v_{k-1}\right)=3$. In that case, we can either have $\ell\left(v_{0} u\right)=1$ in which case $c_{\ell}\left(v_{0}\right)=5$, or $\ell\left(v_{0} u\right)=2$ in which case $c_{\ell}\left(v_{0}\right)=6$.

- If $\ell\left(v_{0} v_{1}\right)=1$ and $\ell\left(v_{0} v_{k-1}\right)=2$, then $\ell\left(v_{1} v_{2}\right)=1$ and $\ell\left(v_{k-1} v_{k-2}\right)=2$, and $c_{\ell}\left(v_{1}\right)=2$ and $c_{\ell}\left(v_{k-1}\right)=4$. In that case, so that $c_{\ell}\left(v_{0}\right) \neq 4$, we must have $\ell\left(v_{0} u\right)=2$ in which case $c_{\ell}\left(v_{0}\right)=5$.

This concludes the proof.

Let $G=H(x, y)$, and $\ell$ be a proper 2-labelling of $G$. Let $H_{1}, H_{2}$ be the two connected components resulting from the removal of the unique bridge $u v$ of $G$, and $G_{1}$ and $G_{2}$ be the subgraphs $H_{1}+u v$ and $H_{2}+u v$, respectively, of $G$ (where, say, $G_{1}$ contains the cycle $C_{1}$ with length $x$, and $G_{2}$ contains the cycle $C_{2}$ with length $y$ ). Applying Claim 5.10 onto $G_{1}$ and $G_{2}$ and the restriction of $\ell$ to these graphs, we deduce that we cannot have $\ell(u v)=1$ as otherwise we would have $c_{\ell}(u)=c_{\ell}(v)=5$, a contradiction. So we must have $\ell(u v)=2$. Furthermore, still by Claim 5.10 exactly half of the edges of $C_{1}$ must be labelled 2 by $\ell$, and similarly exactly half of the edges of $C_{2}$ must be labelled 2. It yields that $\sigma(\ell)=\left\lceil\frac{3}{2}|E(H(x, y))|\right\rceil$. Note that $\ell$ does exist, since $G$ is not an odd multi-cactus (due to the presence of the bridge $u v$ ). In particular, the edges of $C_{1}$ and $C_{2}$ can be labelled in such a way that $c_{\ell}(u)$ and $c_{\ell}(v)$ are two distinct values in $\{4,5,6\}$.

\subsubsection{Improved upper bounds}

As shown previously, it seems that, in general, for nice bipartite graphs the bound in Theorem 5.6 might not be optimal. Following our investigations in the previous section, we believe that perhaps the following could be the right direction to investigate:

Conjecture 5.11. There is an absolute constant $c \geq 1$ such that, for every nice bipartite graph $G \in \mathcal{B}_{2}$, we have $\mathrm{mE}_{2}(G) \leq \frac{3}{2}|E(G)|+c$. 
It is worth pointing out that a proper 2-labelling $\ell$ of a graph $G$ where $\sigma(\ell)$ is about $\frac{3}{2}|E(G)|$ is actually a 2-labelling where the number of assigned 1's is about the same as the number of assigned 2's. Thus, Conjecture 5.11 can be sort of related to the notion of equitable proper labellings of graphs, which were introduced in [3], which are proper labellings where, for every two assigned labels $i, j$, the number of edges assigned label $i$ differs by at most 1 from the number of edges assigned label $j$. Regarding Conjecture 5.11, it can be observed that $\operatorname{mE}_{2}(G) \leq \frac{3}{2}|E(G)|+1$ holds for every graph $G$ admitting an equitable proper 2-labelling. The additive $c$ term is naturally reduced from 1 to 0 when $G$ has even size. Among other results, the authors in 3] proved that nice bipartite complete graphs and forests admit equitable proper 2-labellings.

Towards Conjecture 5.11, in this section our aim is to improve Theorem 5.6 further for the bipartite graphs of $\mathcal{B}_{2}$. First off, we point out that the theoretical upper bound in Theorem 5.6 cannot be reached for a bipartite graph in $\mathcal{B}_{2}$.

Observation 5.12. $\forall G \in \mathcal{B}_{2}, \mathrm{mE}_{2}(G)<2|E(G)|$

Proof. By definition of $\mathcal{B}_{1}, \mathcal{B}_{2}$ and $\mathcal{B}_{3}$, since $G \notin \mathcal{B}_{1}$ the graph $G$ is not locally irregular. Now, if $\mathrm{mE}_{2}(G)=2|E(G)|$, then the only proper 2-labelling of $G$ is the one assigning label 2 to all edges. For such a labelling to be proper, $G$ must have no two adjacent vertices having the same degree. So $G$ must be locally irregular, a contradiction.

In particular contexts, better bounds can be obtained by adapting the arguments from the proof of Theorem 5.3 in a particular way.

Theorem 5.13. Let $G$ be a nice bipartite graph with bipartition $(U, V)$ where $|U|$ is even. Then, we have $\mathrm{mE}_{2}(G) \leq|E(G)|+|V(G)|-1$.

Proof. Let us denote by $U_{e}\left(U_{o}\right.$, respectively) the set of vertices of $U$ having even (odd, respectively) degree in $G$, and similarly by $V_{e}$ ( $V_{o}$, respectively) the set of vertices of $V$ having even (odd, respectively) degree in $G$. Note that either $\left|U_{e}\right|$ and $\left|V_{o}\right|$ must have the same parity, or $\left|U_{o}\right|$ and $\left|V_{e}\right|$ must have the same parity. This is because, otherwise, since $|U|$ is even and $|U|=\left|U_{e}\right|+\left|U_{o}\right|$, the sizes $\left|U_{e}\right|$ and $\left|U_{o}\right|$ must have the same parity, we would get that also $\left|V_{e}\right|$ and $\left|V_{o}\right|$ have the same parity. From this, we would deduce that $\sum_{u \in U} d(u) \not \equiv \sum_{v \in V} d(v)(\bmod 2)$, which is not possible.

Without loss of generality, we may assume that $U_{e}$ and $V_{o}$ have the same parity, thus that $\left|U_{e}\right|+\left|V_{o}\right|$ is even. Our aim now, is to design a 2-labelling of $G$ where all vertices in $U$ get odd colour while all vertices in $V$ get even colour. Such a labelling will obviously be proper. To that aim, we proceed as follows. Let us start with assigning label 1 to all edges of $G$. This way, at this point the colour of every vertex is exactly its degree; so all vertices in $U_{o}$ and $V_{e}$ verify the desired colour property, while all vertices in $U_{e}$ and $V_{o}$ do not. To fix these vertices, we consider any spanning tree $T$ of $G$. We now repeatedly apply the following fixing procedure: we consider any two vertices $x$ and $y$ of $U_{e} \cup V_{o}$ that remain to be fixed, and flip (i.e., turn the 1's into 2's, and vice versa) the labels of all edges on the unique path in $T$ from $x$ to $y$. This way, note that only the colours of $x$ and $y$ are altered modulo 2. Since $\left|U_{e}\right|+\left|V_{o}\right|$ is even, there are an even number of vertices to fix, and, by flipping labels along paths of $T$, we can fix the colour of all vertices in $U_{e} \cup V_{o}$. This results in a 2-labelling $\ell$ of $G$, with the desired properties, which is thus proper.

Note now that $\ell$ assigns label 2 to edges of $T$ only. Since $T$ has $|V(G)|-1$ edges, the result follows.

Note for instance that, for a graph $G$, we have $|E(G)|+|V(G)|-1 \leq \frac{3}{2}|E(G)|$ as soon as $|E(G)| \geq 2|V(G)|-2$. As notable consequences, this implies that a bipartite graph $G \in \mathcal{B}_{2}$ with a part of even size verifies $\mathrm{mE}_{2}(G) \leq \frac{3}{2}|E(G)|$ as soon as $G$ has minimum degree at least 4 , or more generally when the graph is dense enough.

The same result also holds when $G$ is bipartite and cubic (in which case $\chi_{\Sigma}(G)=2$, by definition of odd multi-cacti), from a more general argument:

Observation 5.14. Let $G$ be a regular graph with $\chi_{\Sigma}(G)=2$. Then, we have $\mathrm{mE}(G) \leq \mathrm{mE}_{2}(G) \leq$ $\frac{3}{2}|E(G)|$. 
Proof. Let $\ell$ be a proper 2-labelling of $G$. Since $G$ is regular, the edges labelled 1 by $\ell$, and similarly the edges labelled 2, must induce a locally irregular subgraph of $G$. Then the 2-labelling $\ell^{\prime}$ of $G$ obtained by turning all 1's into 2's, and vice versa, is also proper. Now there is one of $\ell$ and $\ell^{\prime}$ that assigns label 2 to at most half of the edges, and the conclusion follows.

Slight modifications of the proof of Theorem 5.13 also yields the desired result for certain bipartite graphs that are Hamiltonian.

Observation 5.15. Let $G$ be a Hamiltonian bipartite graph with bipartition $(U, V)$ where $|U|$ is even. Then, we have $\mathrm{mE}(G) \leq \mathrm{mE}_{2}(G) \leq \frac{3}{2}|E(G)|$.

Proof. Just mimic the proof of Theorem [5.13, but repair pairs of defective vertices of $G$ along a Hamiltonian cycle $C=\left(v_{0}, \ldots, v_{n-1}, v_{0}\right)$, matching each of them, say, with the next defective vertex in the ordering of $C$. If this fixing process turns more than half of the edges to 2 , then, instead, repair pairs of vertices around $C$ matching each of them with the previous defective vertex in the ordering (which is equivalent to flipping the labels along $C$ ).

\subsection{Trees}

Our main result here is that for every nice tree $T$, we have $\mathrm{mE}_{2}(T) \leq \frac{3}{2}|E(T)|$, which cannot be lowered in general, due to Corollary 5.8. Still, it confirms Conjecture 5.11 for nice trees. Let us recall that it was proved in [3] that nice forests admit equitable proper 2-labelling. This directly implies our result below for trees with even size, while it does not for trees with odd size (as a 2-labelling where the number of assigned 2's is one more than the number of assigned 1's does not fulfil our claim).

Theorem 5.16. For every nice tree $T$, we have $\mathrm{mE}_{2}(T) \leq \frac{3}{2}|E(T)|$.

Proof. The proof is by induction on the number of branching vertices of $T$. The base case is when $T$ has no branching vertex, i.e., when $\Delta(T) \leq 2$. In that case, $T$ is a path. Let us here consider the 2-labelling $\ell$ of $T$ obtained by assigning labels $1,1,2,2,1,1,2,2, \ldots$ as traversing the edges from an end-vertex to the second one. It is easy to see that $\ell$ is indeed proper. Indeed, recall first that degree-1 vertices cannot be involved in a colour conflict. Furthermore, for two adjacent 2-vertices $u, v$ to have the same colour, the edge incident to $u$ different from $u v$ must be assigned the same label as the edge incident to $v$ different from $u v$ (recall Observation 2.3). Since, by $\ell$, no two edges at distance 2 get assigned the same label, this implies that no colour conflict arises. Lastly, since 1 's and 2's are assigned by pairs starting from a pair of 1's, it should be clear that $\ell$ assigns more 1's than 2's. Thus, $\mathrm{mE}_{2}(T) \leq \sigma(\ell) \leq \frac{3}{2}|E(T)|$.

We now focus on the general case. That is, we now assume that $T$ has branching vertices, and every nice tree with fewer branching vertices verifies the claim. Let us root $T$ at some degree-1 vertex $r$. In the usual way, this defines a (virtual) orientation of $T$, where every vertex $v$ of $T$ but $r$ has a unique parent (unique neighbour of $v$ that is closer to $r$ ), all vertices $v$ but the leaves have children (neighbours of $v$ that are farther from $r$ ), and more generally all vertices $v$ but the leaves have descendants (vertices where their unique shortest path to $r$ passes through $v$ ). By a deepest branching vertex of $T$, we refer to a branching vertex whose all descendants are not branching vertices, i.e., they have degree at most 2.

Let us consider a deepest branching vertex $v$ of $T$. Then $v$ is adjacent to its parent $w$ and there are $k \geq 2$ hanging paths $P_{1}, \ldots, P_{k}$ attached to $v$. Note that some of the $P_{i}$ 's may be of length 1 in case some of the children of $T$ are leaves. Let $T^{\prime}$ be the tree obtained from $T$ by removing the edges of $P_{1}, \ldots, P_{k}$ (i.e., all their vertices different from $v$ ). If $T^{\prime}$ is just an edge, then $T$ is actually a subdivided star. If $T$ is a star with at least two leaves, then it is locally irregular and we can assign label 1 to all edges. Otherwise, when $T$ is a subdivided star different from a star, then, without loss of generality, $P_{1}$ has length at least 2 . We then change the root $r$ to be the degree-1 vertex of $P_{1}$ so that, now, we can assume that $T^{\prime}$ indeed is not an edge.

Thus, we can assume that $T^{\prime}$ is not just an edge. Since $T^{\prime}$ has less branching vertices than $T$, by the induction hypothesis there is a 2-labelling $\ell^{\prime}$ of $T^{\prime}$ verifying $\mathrm{mE}\left(T^{\prime}, \ell^{\prime}\right) \leq \frac{3}{2}\left|E\left(T^{\prime}\right)\right|$. We wish to extend $\ell^{\prime}$ to the edges of $P_{1}, \ldots, P_{k}$, thus to a 2-labelling $\ell$ of $T$. To that aim, we consider the following two extension schemes for extending $\ell^{\prime}$ to the edges of one $P_{x}$ of the $P_{i}$ 's: 
- 1-extension: We assign labels $1,1,2,2,1,1,2,2, \ldots$ to the consecutive edges of $P_{x}$, as they are traversed going from $v$ to the degree- 1 vertex of $P_{x}$.

- 2-extension: We assign labels $2,1,1,2,2,1,1,2,2, \ldots$ to the consecutive edges of $P_{x}$, as they are traversed going from $v$ to the degree- 1 vertex of $P_{x}$.

Note that whenever $P_{x}$ has length not congruent to 1 modulo 4, the number of assigned 1's is always at least the number of assigned 2's by both 1-extensions and 2-extensions. More precisely, if $P_{x}$ has length congruent to 1 modulo 4, then the number of 2 's by a 2-extension is one more than the number of 1's, and vice versa by a 1-extension. Recall also that 1-vertices cannot be involved in colour conflicts. Furthermore, for two adjacent 2 -vertices $x, y$ to have the same colour, the edge incident to $x$ different from $x y$ must be labelled the same way as the edge incident to $y$ different from $x y$. From this, we deduce that when extending $\ell^{\prime}$ to the edges of the $P_{i}$ 's via 1-extensions and 2-extensions, we must just make sure that 1) the colour of $v$ does not get equal to the colour of its parent $w$, and 2) the colour of $v$ does not get equal to the colour of one of its children.

We note that the second type of colour conflict cannot actually occur. Indeed, note that by a 1-extension of $P_{x}$, the neighbour of $v$ in $P_{x}$, unless it has degree 1 (in which case it cannot be in conflict with $v$ ), gets the colour 2, while, by a 2 -extension, it gets the colour 3 . Since $v$ is a branching vertex with $k \geq 2$ children, thus of degree $k+1$, when performing 1-extensions and 2-extensions to the $P_{i}$ 's, vertex $v$ gets colour precisely $k+1 \geq 3$ if only 1-extensions are performed, and colour at least $k+2 \geq 4$ if at least one 2-extension is performed.

Thus, we just need to find a combination of 1-extensions and 2-extensions to the $P_{i}$ 's so that no colour conflict involving $v$ and its parent $w$ arises. Also, we need to make sure that the number of assigned 1's is at least the number of assigned 2's. If one of the $P_{i}$ 's has length not congruent to 1 modulo 4 , then we choose it as $P_{1}$. Otherwise, if they all have length congruent to 1 modulo 4, then we choose any $P_{i}$ as $P_{1}$.

We first perform 1-extensions only, i.e., to all $P_{i}$ 's. If the colour of $v$ gets different from that of $w$, then we are done. Otherwise, when performing a 2-extension to $P_{1}$ and a 1-extension to all other $P_{i}$ 's, the colour of $v$ gets bigger, thus getting different from the colour of $w$. This results in the desired extension $\ell$ to all edges of $T$.

Let us conclude by noting that the number of 1 's assigned by $\ell$ is at least the number of assigned 2's. This is because $\mathrm{mE}_{2}\left(T^{\prime}, \ell^{\prime}\right) \leq \frac{3}{2}\left|E\left(T^{\prime}\right)\right|$, and, as mentioned earlier, by 1-extensions to the $P_{i}$ 's the number of assigned 1's is at least the number of assigned 2's. By 2-extensions, this is true when performed on paths of length not congruent to 1 modulo 4 . By our choice of $P_{1}$, if $P_{1}$ has length congruent to 1 modulo 4 , then so do all $P_{i}$ 's. In that precise case, the number of 2 's assigned to the edges of $P_{1}$ is one bigger than the number of assigned 1's, but this is compensated by the fact that, in $P_{2}$, the number of assigned 1's is one bigger than the number of assigned 2's. Thus we additionally have $\sigma(\ell) \leq \frac{3}{2}|E(T)|$, as desired.

\section{Conclusion}

In this work, we have studied proper labellings of graphs with the additional requirement that we want the sum of assigned labels to be as small as possible. Our interests were guided by both straight questions, such as determining $\operatorname{mE}(G)$ for a given graph $G$, as well as more fundamental ones, such as the difference, in general, between $\mathrm{mE}_{k}(G)$ and $\mathrm{mE}_{k^{\prime}}(G)$ for $k \neq k^{\prime}$. We have also investigated the complexity of finding "optimal" labellings.

We quickly ran into Conjecture 5.2, which seems rather natural when knowing that the 1-2-3 Conjecture seems to be a more than plausible conjecture and that graphs, in general, seem to need only a few 3's to design proper 3-labellings. We believe it would be interesting to further study this point in the future, as Conjecture 5.2 stands as the main open problem regarding our investigations in the current paper. It would also be interesting to progress towards its refinement for bipartite graphs, Conjecture 5.11. A way to progress towards answering both questions could be to exhibit families of (possibly bipartite) graphs $G$ for which $\mathrm{mE}(G)$ is "large", i.e. larger than the quantity in Theorem 5.9.

Regarding our algorithmic results in Section 3, we note that they all deal, for a given graph $G$, with the parameter $\operatorname{mE}_{k}(G)$ (for some $k$ ), and not with the more general parameter $\mathrm{mE}(G)$. 
This is mainly because, as indicated by Theorem 4.5, in general there is no absolute constant that bounds, for all graphs $G$, the smallest $k$ such that $\mathrm{mE}(G)=\mathrm{mE}_{k}(G)$. In particular, even for a graph $G$ of bounded treewidth, although we can determine $\mathrm{mE}_{k}(G)$ in polynomial time for any $k$ (due to our algorithm in Theorem 3.5), running multiple iterations of our algorithm to determine $\mathrm{mE}(G)$ is not feasible in polynomial time. Thus, we leave the following problem open even for the simplest case:

Question 6.1. What is the complexity of determining $\mathrm{mE}(T)$ for a given tree $T$ ?

\section{References}

[1] O. Baudon, J. Bensmail, H. Hocquard, M. Senhaji, É. Sopena. Edge Weights and Vertex Colours: Minimizing Sum Count. Discrete Applied Mathematics, 270:13-24, 2019.

[2] O. Baudon, J. Bensmail, J. Przybyło, M. Woźniak. On decomposing regular graphs into locally irregular subgraphs. European Journal of Combinatorics, 49:90-104, 2015.

[3] O. Baudon, M. Pilśniak, J. Przybyło, M. Senhaji, É. Sopena, M. Woźniak. Equitable neighbour-sum-distinguishing edge and total colourings. Discrete Applied Mathematics, 222:40-53, 2017.

[4] J. Bensmail, F. Mc Inerney, K. Szabo Lyngsie. On $\{a, b\}$-edge-weightings of bipartite graphs with odd $a, b$. Accepted for publication in Discussiones Mathematicae Graph Theory.

[5] J. Bensmail, B. Li, B. Li, N. Nisse. On Minimizing the Maximum Color for the 1-2-3 Conjecture. Technical report, 2019. Available online at https://hal.archives-ouvertes.fr/ hal-02330418.

[6] H.L. Bodlaender. A partial $k$-arboretum of graphs with bounded treewidth. Theoretical Computer Science, 209(1-2):1-45, 1998.

[7] G.J. Chang, C. Lu, J. Wu, Q. Yu. Vertex-coloring edge-weightings of graphs. Taiwanese Journal of Mathematics, 15(4):1807-1813, 2011.

[8] G. Chartrand, P. Erdös, O.R. Oellermann. How to define an irregular graph. College Mathematics Journal, Vol. 19, No 1, 1988.

[9] G. Chartrand, M.S. Jacobson, J. Lehel, O.R. Oellermann, S. Ruiz, F. Saba. Irregular networks. Congressus Numerantium, 64:197-210, 1988.

[10] A. Dudek, D. Wajc. On the complexity of verterx-coloring edge-weightings. Discrete Mathematics Theoretical Computer Science, 13(3):45-50, 2011.

[11] M. Kalkowski, M. Karoński, F. Pfender. Vertex-coloring edge-weightings: towards the 1-2-3 Conjecture. Journal of Combinatorial Theory, Series B, 100:347-349, 2010.

[12] M. Karoński, T. Łuczak, A. Thomason. Edge weights and vertex colours. Journal of Combinatorial Theory, Series B, 91:151-157, 2004.

[13] H. Lu, Q. Yu, C.-Q. Zhang. Vertex-colouring 2-edge-weightings of graphs. European Journal of Combinatorics, 32:21-27, 2011.

[14] W. Mulzer, G. Rote. Minimum-weight triangulation is NP-hard. Journal of the ACM, 55(2):11, 2008.

[15] J. Przybyło. The 1-2-3 Conjecture almost holds for regular graphs. Preprint, 2018. Available online at https://arxiv.org/abs/1809.10761

[16] B. Seamone. The 1-2-3 Conjecture and related problems: a survey. Preprint, 2012. Available online at http://arxiv.org/abs/1211.5122. 
[17] K. Szabo Lyngsie. On neighbour sum-distinguishing $\{0,1\}$-weightings of bipartite graphs. Discrete Mathematics and Theoretical Computer Science, 20(1), 2018, \#21.

[18] C. Thomassen, Y. Wu, C.-Q. Zhang. The 3-flow conjecture, factors modulo $k$, and the 1-2-3conjecture. Journal of Combinatorial Theory, Series B, 121:308-325, 2016. 